\title{
Towards a Visual Simultaneous Localization and Mapping system for computationally constrained systems
}

\author{
by \\ Pablo Roberto Molina Cabrera, B.Sc. \\ A thesis submitted to the \\ Faculty of Graduate and Postdoctoral Affairs \\ in partial fulfillment of the requirements for the degree of
}

Master of Applied Science in Mechanical and Aerospace Engineering

Ottawa-Carleton Institute for Electrical and Computer Engineering

Department of Mechanical and Aerospace Engineering

Carleton University

Ottawa, Ontario

May, 2015

(C) Copyright

Pablo Roberto Molina Cabrera, 2015 
The undersigned hereby recommends to the

Faculty of Graduate and Postdoctoral Affairs acceptance of the thesis

\title{
Towards a Visual Simultaneous Localization and Mapping system for computationally constrained systems
}

\author{
submitted by Pablo Roberto Molina Cabrera, B.Sc. \\ in partial fulfillment of the requirements for the degree of \\ Master of Applied Science in Mechanical and Aerospace Engineering \\ Professor Alex Ellery, Supervisor \\ Professor Jurek Sasiadek, Examiner \\ Professor Claire Samson, Internal Examiner \\ Professor Natalie Baddour, External Examiner \\ Professor Metin I. Yaras, Chair, \\ Department of Mechanical and Aerospace Engineering
}


Ottawa-Carleton Institute for Electrical and Computer Engineering Department of Mechanical and Aerospace Engineering

\section{Carleton University}

May, 2015 


\section{Abstract}

Due to the lack of global positioning systems on space exploration missions, robotic exploration missions such as the Mars Exploration Rovers (MER) had to rely on relative localization techniques such as visual odometry ( $\mathrm{VO}$ ) to estimate the robot position in an unknown environment. Unfortunately, the error of VO grows superlinearly with the distance travelled. Future robotic planetary exploration missions such as sample retrieval and in-situ resource utilization will require more accurate localization techniques such as Simultaneous Localization and Mapping (SLAM) to reduce the error in motion estimation and achieve the science goals. One of the main reasons why SLAM techniques are not being used in robotic exploration missions is their computational cost. The computer systems on-board exploration robots have limited processing power that is often used for other maintenance tasks such as communications, power management, etc. In this thesis, a visual SLAM system aimed towards computationally constrained systems is presented. The approach described in this thesis uses only a RGB-D sensor as input to the system. Most visual SLAM systems require computationally intensive feature descriptors such as Speeded Up Robust Features (SURF) and Scale-Invariant Feature Transform (SIFT) as part of their front-end SLAM system. In this work, Binary Robust Invariant Scalable Keypoints (BRISK) and Oriented FAST and Rotated BRIEF (ORB) feature descriptors are introduced and compared against SURF. BRISK is shown to achieve similar relative pose estimation performance than SURF while being an order of magnitude faster. In addition, this work also discusses a simple back-end pose-graph optimization approach using $l i b g^{2} o$. The back end system improved the position estimation as well as detected loop closure events. The algorithm was tested with an indoor dataset as well as publicly available robot dataset. These initial results show that computationally inexpensive feature detectors such as BRISK and ORB can be used as core feature detection algorithms for a visual SLAM system. 


\section{Acknowledgments}

I would like to extend my deepest gratitude to Dr. Alex Ellery for his guidance and support throughout my studies and during this thesis. His support was fundamental for the completion of this work.

I would like to thank all the members of the Space Exploration Engineering Group whom helped me in several ways while working on this thesis.

To my loving girlfriend, for her love, undying support and kind words of encouragement, I am truly thankful.

Finally, I would like to thank my parents for their constant support, encouragement and inspiration to complete this thesis. Without their constant encouragement, I will not be where I am today. ("Gracias por todo chaseitos") 


\section{Table of Contents}

Abstract $\quad$ iv

Acknowledgments $\quad$ v

Table of Contents vi vi v

List of Tables viii

List of Figures $\quad$ ix

Nomenclature $\quad$ xi

1 Introduction 1

1.1 Introduction . . . . . . . . . . . . . . . . 1

1.1.1 The problem ..................... 1

1.1.2 High-level overview .................. 4

2 Literature Review 5

2.1 Related work . . . . . . . . . . . . . . . 5

2.2 Front-end system (visual odometry) . . . . . . . . . . 5

2.2.1 Camera calibration ............... 6

2.2.2 Feature detection and description . . . . . . . . . . . 9

2.2.3 Feature matching . . . . . . . . . . . . . . 13

2.2.4 Outlier Rejection .................. 14

2.2.5 Motion Estimation . . . . . . . . . . . . . . . 15

2.2.6 Other front-end approaches . . . . . . . . . . . 15

2.3 Back-end system . . . . . . . . . . . . . . . . 16

2.3.1 Bundle adjustment . . . . . . . . . . . . . . . 17 
2.3.2 Graph SLAM . . . . . . . . . . . . . . . . 24

3 Approach $\quad 25$

3.1 Proposed Approach . . . . . . . . . . . . . . . . . . . . 25

3.1.1 System Overview . . . . . . . . . . . . . . 25

3.1.2 Thesis requirements and implementation overview . . . . . . . 26

3.1.3 Kinect sensor calibration . . . . . . . . . . . . . . 27

3.1.4 Front End System . . . . . . . . . . . . . . . . . . . 31

3.1.5 Graph SLAM (back end) . . . . . . . . . . . . . . . 37

4 Experiments $\quad 41$

4.1 Experiments . . . . . . . . . . . . . . . . 41

4.1.1 Implementation details . . . . . . . . . . . . . . . . 44

4.1.2 Kinect Sensor Calibration . . . . . . . . . . . . . . . 44

4.1.3 Feature detection/descriptor timing comparison . . . . . . . 45

4.1 .4 Matching results . . . . . . . . . . . . . . 47

4.1.5 Outlier Rejection and transformation refinement performance 48

4.1.6 Back end performance . . . . . . . . . . . . . . . . 51

5 Discussion $\quad 58$

5.1 Results discussion . . . . . . . . . . . . . . . . . 58

5.1 .1 Front-end discussion ................. 58

5.1 .2 Back-end discussion . . . . . . . . . . . . . . 60

5.1 .3 Future Work . . . . . . . . . . . . . . . . . . 60

6 Conclusion $\quad 62$

6.1 Conclusion . . . . . . . . . . . . . . . . . 62

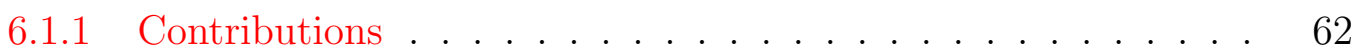

$\begin{array}{ll}\text { List of References } & 64\end{array}$

Appendix A Sample images from Indoor Carleton Dataset $\quad 71$ 


\section{List of Tables}

2.1 Comparison of feature detector algorithms. Computer system employed is a laptop with Core i7-2720QM @ 2.2Ghz, 8Gb of RAM . . . 12

4.1 Calibration parameters for the infra-red camera RGB camera of the Kinect sensor used in the indoor dataset . . . . . . . . . . . . . . . . 45

4.2 The effect of introducing the Minimum Distance Multiplier (MinDistMultiplier) when finding the top matches for BRISK and ORB feature description algorithms (Carleton indoor dataset) . . . . . . . . .

4.3 The effect of introducing the Minimum Distance Multiplier (MinDistMultiplier) when finding the top matches for BRISK and ORB feature description algorithms (Freidburg dataset) . . . . . . . . . . .

4.4 Accuracy results for the front end system organized by feature descriptor and algorithm variations (Carleton Indoor dataset) . . . . . . . .

4.5 Accuracy results for the front end system organized by feature descriptor and algorithm variations (Freidburg dataset) . . . . . . . . . .

4.6 Back end SLAM system results organized by feature description algorithm used (Carleton indoor dataset) . . . . . . . . . . . . .

4.7 System results organized by feature description algorithm used (Freidburg SLAM dataset) . . . . . . . . . . . . . . . . 


\section{List of Figures}

2.1 Visual Odometry modern processing pipeline . . . . . . . . . 6

2.2 Camera models - (a) Perspective projection (pin-hole model), Let $X=$ $[x, y, z]$ be a scene point in the camera reference frame and $p=[u, v]$ its projection on the image plane measured in pixels. . . . . . . 7

2.3 Bundle adjustment frame notation. . . . . . . . . . . . . . 18

2.4 Sparse structure of the $A$ and $B$ Jacobians for a bundle adjustment problem of three poses and four features. . . . . . . . . . . 23

3.1 System overview diagram . . . . . . . . . . . . . . . . 25

3.2 Kinect sensor infrared depth sensing. a) Infrared image of the pattern of speckles projected on a sample scene. b) The resulting depth image

3.3 Illustration of the Microsoft kinect optical axis and how depth is mea-

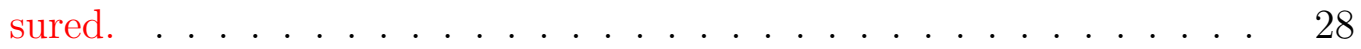

3.4 a) RGB image of a chair b) Depth image of the same chair. A shadow image on the left of the chair (depth equal to 0). This occurs due to the relative position of the infra-red emitter and the infra-red camera.

4.1 Sample image from the office testing environment. The green arrows represent the optical flow generated with the final matches once RANSAC has removed outliers. (the feature detection/descriptor algorithm used was SURF) . . . . . . . . . . . . . . . 41

4.2 Carleton Indoor Dataset overview. The viewpoints are illustrated by arrows and the sequence by numbers $\ldots \ldots \ldots \ldots$

4.3 Sample image from the Freidburg dataset. The green arrows represent the optical flow generated with the final matches once RANSAC has removed outliers. (the feature detection/descriptor algorithm used was

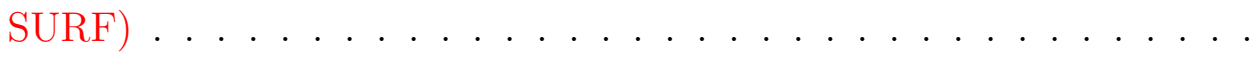

4.4 Box plot of feature descriptor and detection time for SURF, BRISK and ORB algorithms (Carleton indoor dataset) $\ldots \ldots \ldots \ldots$ 
4.5 Processing time for each frame arranged by feature detection/descriptor algorithm used. . . . . . . . . . . . . . . . 50

4.6 Processing time for the back end graph optimization arranged by image number (Carleton indoor Dataset) . . . . . . . . . . . . .

4.7 Processing time for the back end graph optimization arranged by image number (Freidburg Dataset) . . . . . . . . . . . . . . . . .

4.8 Results of applying our approach on the Freidburg SLAM dataset. (The BRISK feature detector was employed) . . . . . . . . . . .

4.9 Results of applying our approach on the Freidburg SLAM dataset. (The SURF feature detector was employed) . . . . . . . . . . . 56

5.1 Visual Odometry Error measured during a 2.45 meter drive using HAZCAMs on the MER Surface System Testbed Lite rover. . . . . . . . . 


\section{Nomenclature}

\begin{tabular}{cr}
\hline Symbol & Description \\
\hline$x^{\prime}$ & Camera Vector containing pixel coordinates $(\mathrm{u}, \mathrm{v})$ \\
$f x, f y$ & Intrinsic camera calibration parameter matrix \\
$c x, c y$ & Camera Focal length in pixel units for $\mathrm{x}$ and $\mathrm{y}$ \\
$X^{\prime}$ & Camera Principal point for $\mathrm{x}$ and $\mathrm{y}$ \\
$F_{0}$ & The global inertial frame \\
$F_{k}$ & Crame attached to the robot at time $k$ \\
$x_{k}$ & Thector representing the pose of the robot at time $\mathrm{k}$ \\
$p_{0}^{j, 0}$ & The position of a feature $j$ in the global inertial frame \\
$z_{k, j}$ & The measurement of feature $j$ at time $k$ \\
\hline
\end{tabular}




\section{Glossary}

BRISK Binary Robust Invariant Scalable Keypoints. ix, 1, 2, 7, 13-15, 21, 24, 27

EKF Extended Kalman Filter. ix, 11

FAST Features from Accelerated Segment Test. ix, 11, 13

ICP Iterative Closest Point. ix, 11

IMU Intertial Measurement Unit. ix, 3, 12

MCL Monte Carlo Localisation. ix

ORB Oriented FAST and Rotated BRIEF. ix, 1, 2, 13-15, 21, 24, 27

RANSAC Random Random Sample Consensus. ix, 9, 10, 14-17, 21-24

SIFT Scale-Invariant Feature Transform. ix, 1, 11, 13

SLAM Simultaneous Localization and Mapping. ix, 1, 3, 4, 11-13

SURF Speeded Up Robust Features. ix, 1, 2, 11, 13-15, 21, 24 


\section{Chapter 1}

\section{Introduction}

\section{$1.1 \quad$ Introduction}

\subsubsection{The problem}

In large environments lacking a global positioning system (GPS), accurate robot navigation is a difficult problem. The Mars Exploration Rovers (MERs) have been in the surface of Mars since 2004 [1]. Detailed human-generated motion commands are sent to the robots generally once every Martian sol (a martian solar day - duration of 24 hours and 39 minutes) [2]. The human-generated commands maximize safety and scientific return of the mission. The delay in the communication and the humanin-the-loop control scheme limits the speed of the robots to generally less than 40 meters per Martian sol [2].

The MERs use a relative localization system to accurately execute the control commands. The relative localization system uses wheel odometry, Inertial Measurement Unit (IMU) and a visual odometry algorithm. Since the terrain in Mars can have steep slopes with sand and small stones, the visual odometry algorithm is essential to detect wheel slip and hence correct the position estimates [1]. However, visual odometry estimates also accumulate errors with super-linear growth in the distance travelled, owing to increasing orientation errors [3].

There are several newly planned missions that will require long-range autonomous operation of rovers [4]. New missions are being planned for "sample-and-return" as well as In-Situ resource utilization (ISRU) [5]. Employing relative localization systems with super-linear error growth does not provide enough accuracy for longrange autonomy [6]. 
One solution to improve the localization of robots is to use Simultaneous Localization and Mapping (SLAM) in the working area. Visual "teach-and-repeat" (VT\& R) has proven to be an effective technique for solving the sample return task. VT\& $\mathrm{R}$ employs a version of visual SLAM to construct a series of maps of the area during the "teach" pass and stores this maps in memory. The "repeat" pass is completed by referencing these maps and comparing the current views with the previously seen views in order to "repeat" the taught route [6]. Along with VT \& R, there are several visual SLAM systems available in the literature. [7] [8] [9] [10] [11].

While visual SLAM is a proven technique for improving localization, most of the work in visual SLAM employs computationally-intensive operations such as feature detection/description (Scale-Invariant Feature Transform (SIFT) and Speeded Up Robust Features (SURF)) and versions of bundle-adjustment [6] [7] [8]. Some visual SLAM algorithms are designed to run on real-time on small commercial-grade computers [12] [11]. However, the aforementioned real-time algorithms are limited to small areas and do not scale to larger areas such as those encountered in exploration missions.

\section{Computer systems in robotic exploration missions}

Computer systems are an integral part of all current spacecraft. Computers are being used for navigation functions such as rendezvous, re-entry, and mid-course corrections. In addition, computers are used for system management functions, data formatting, attitude control, etc [13]. Most spacecraft computer systems are designed to operate in "real-time" mode handling essentially asynchronous inputs and outputs and continuous processing. This "real-time" requirement leads to other requirements for spacecraft computers not normally found on commercial earth-based systems. The software running on board spacecraft must not "crash" at all or have an abrupt end. If the software running on the spacecraft stops, the vehicle cannot be controlled any more [14]. The hardware must also be hardened against radiation and temperature swings experienced in space travel. In fact, every computer system used in spacecraft must pass the MIL-STD-883E certification. This certification requires the computer system to pass more than one hundred different tests on mechanical, thermal, AC electrical, DC electrical as well as radiation and individual wafer inspections. [15].

Due to the aforementioned requirements, computer systems in spacecraft often 
lag behind the state-of-the-art earth-based systems. Spacecraft computer systems often use thoroughly tested Computer Processing Units (CPU) that are known to work reliable for several years. Once the $\mathrm{CPU}$ is chosen, the process of hardening the device begins and takes several years of design and testing [14]. For example, The Mars Exploration Rovers (MERs) which were launched in 2004 used a BAE RAD6000 computer running at 20Mhz. The next Mars mission which was launched in late 2011, the Mars Science Laboratory (MSL), uses two BAE-Systems RAD750 which produces around 400 Million Instructions per second (MIPS) at 200Mhz [4] (one computer for normal use and another for redundancy). The MSL computer system is also in charge of running maintenance tasks such as thermal control, communications etc. Only $75 \%$ of the processing power on the MSL computer system is available for navigation tasks which include path planning, obstacle avoidance and lastly position estimation [4]. As a comparison, the Intel i5-2550K CPU which was released in January 2011 produces around 45000 MIPS at 2500Mhz [16]. Although comparing CPU performance requires more analysis that merely comparing MIPS, it is interesting to see that the i5 mediumgrade commercial CPU can achieve two orders of magnitude more MIPS than the MSL computer system.

The ExoMars European mission also has a limited computing power. ESA has started efforts such as the SPARTAN project in order to move computationally intensive operations away from the main general purpose computer system of the robot. In the SPARTAN project, a 150MIPS FPGA system is suggested as a potential computing system dedicated to SLAM, localization and stereo vision for path planning [17]. It is clear from the examples shown above that if a SLAM system were employed in a future mission, the algorithm must minimize the computational load while keeping the accuracy to acceptable levels.

This thesis describes a full visual SLAM system aimed at computational constrained systems. The choice of feature detector/extractor algorithm, motion estimation and back end algorithm allow the system to run on real-time without needing a graphics processing unit (GPU) or large CPU load. The main sensor employed in this work is the RGB-D camera - Microsoft Kinect. While this RGB-D camera does not operate in outdoor environments [18], stereo cameras such as the PointGrey BumbleBee2 [19] and FLASH-LIDAR systems such as Peregrine 3D-LIDAR [20] can be configured to provide intensity images as well as the depth information of an outdoor scene. 


\subsubsection{High-level overview}

This thesis begins by providing a a literature review on SLAM. Every aspect of the generic visual SLAM pipeline is discussed in detail. An in-depth review of the current state-of-the-art feature detection and description is presented since this step is generally the most computationally expensive step. A review of motion estimation and back-end SLAM systems is presented. Once the literature review is completed, the approach section provides further details regarding the approach followed specifically for this thesis. The approach section includes detailed calibration models for the Kinect camera and a description of the matching and outlier rejection algorithms. After the algorithms are described in depth, a report of the results of the system on real datasets is presented in the experiments section. The experiment section also includes specific implementation details as well as timing and performance results. Finally, the conclusion section summarizes the results and contributions. Recommendations on future work are also included 


\section{Chapter 2}

\section{Literature Review}

\section{$2.1 \quad$ Related work}

The general problem of SLAM has a long history in robotics. A broad survey of all SLAM approaches can be found in [21]. In particular, the field of vision-based SLAM has been heavily studied in the literature. Most visual SLAM systems can be divided in a "front-end" and a "back-end" [22]. The "front-end" system estimates the relative motion between the current frame and previous frames. This relative estimation can be aided by the use of the robot's wheel odometry and IMU. The "back-end" system generally reduces accumulated error by constructing a pose-graph of the motion and attempting to achieve "loop closure". Combining the relative transformations to previous frames will increase the camera-pose error with time. However, transformation estimation to much earlier frames (loop-closure) can greatly reduce the accumulated error [23].

The literature review is subdivided in "front-end" review and "back-end" review sections.

\section{$2.2 \quad$ Front-end system (visual odometry)}

The main purpose of the front-end system is to estimate the frame-to-frame motion. The most commonly used front-end system is Visual Odometry. The first original Visual Odometry algorithm was introduced by [24]. This implementation contains the main steps in the modern Visual Odometry processing pipeline: feature detection, feature matching and motion estimation. Based on this initial work, Matthies 


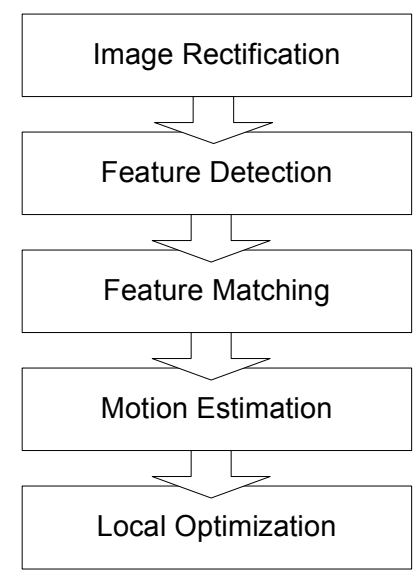

Figure 2.1: Visual Odometry modern processing pipeline

improved the accuracy by formulating the motion estimation step as a statistical estimation problem and modelling landmark uncertainty [25]. The work of [25] is the main algorithm used in the Mars Exploration Rovers [1] which is the main modern visual odometry pipeline. The modern visual odometry pipeline is illustrated in Figure 2.1. The following subsections will provide background on all the steps in the modern visual odometry pipeline.

\subsubsection{Camera calibration}

The main input of visual SLAM systems are images from camera systems. Before images can be used for motion estimation, they must be calibrated. In the scope of this thesis, only the pinhole model (perspective projection) model is analysed although the other models are discussed in depth in this work [26]. An illustration of the pinhole camera model can be seen in Figure 2.2 [22]. 


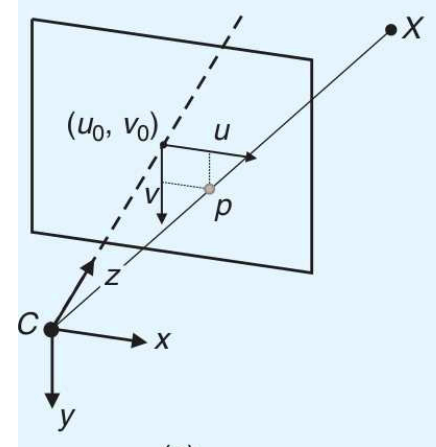

(a)

Figure 2.2: Camera models - (a) Perspective projection (pin-hole model), Let $X=[x, y, z]$ be a scene point in the camera reference frame and $p=[u, v]$ its projection on the image plane measured in pixels.

There are 3 main set of parameters that needs to be calibrated in a pinhole ; intrinsic parameters, extrinsic parameters and lens distortion parameters. The intrinsic and extrinsic parameters can be summarized in Equation 2.1 [27].

$$
\begin{gathered}
s x^{\prime}=A[R \mid t] X^{\prime} \\
s\left(\begin{array}{c}
u \\
v \\
1
\end{array}\right)=\left(\begin{array}{ccc}
f x & 0 & c x \\
0 & f y & c y \\
0 & 0 & 1
\end{array}\right) *\left[\begin{array}{cccc}
r_{11} & r_{12} & r_{13} & t_{1} \\
r_{21} & r_{22} & r_{23} & t_{2} \\
r_{31} & r_{32} & r_{33} & t_{3}
\end{array}\right] *\left(\begin{array}{c}
X \\
Y \\
Z \\
1
\end{array}\right)
\end{gathered}
$$

In the equations above, $x^{\prime}$ is the pixel coordinates $(\mathrm{u}, \mathrm{v}), s$ is the scale (if required), $A$ is the intrinsic calibration parameters which consists of the focal length in pixel units (fx,fy) and the principal point which is the image centre (cx, cy). The matrix $[R \mid t]$ are the extrinsic calibration parameters that are mainly just a homogeneous transform between the camera centre the vehicle centre. The vector $X=(X, Y, Z)$ are the coordinates of the point in $3 \mathrm{D}$ in world coordinate space. We can also define $(x, y, z)$ as the coordinates of the point in camera-frame. Assuming that $z \neq 0$, one 
can write:

$$
\left(\begin{array}{l}
x \\
y \\
z
\end{array}\right)=R\left(\begin{array}{l}
X \\
Y \\
Z
\end{array}\right)+t
$$

Defining $x^{\prime}$ and $y^{\prime}$ as:

$$
\begin{aligned}
x^{\prime} & =\frac{x}{z} \\
y^{\prime} & =\frac{y}{z}
\end{aligned}
$$

Assuming that the homogeneous transformation is the identity, we can write:

$$
\begin{aligned}
& u=f_{x} \dot{x^{\prime}}+c_{x} \\
& v=f_{y} \dot{y}^{\prime}+c_{y}
\end{aligned}
$$

The above equations do not take into consideration the lens distortion. For the purpose of this thesis only the tangential and radial factors are considered. In order to account for the radial distortion Equation 2.8 is introduced.

$$
\begin{aligned}
& x^{\prime \prime}=x^{\prime}\left(1+k_{1} r^{2}+k_{2} r^{4}+k_{3} r^{6}\right) \\
& y^{\prime \prime}=y^{\prime}\left(1+k_{1} r^{2}+k_{2} r^{4}+k_{3} r^{6}\right) \\
& u=f_{x} * x^{\prime \prime}+c_{x} \\
& v=f_{y} * y^{\prime \prime}+c_{y}
\end{aligned}
$$

where $x^{\prime \prime}$ and $y^{\prime \prime}$ are the corrected versions of $x^{\prime}$ and $y^{\prime}$ respectively, the parameters $k_{1}, k_{2}, k_{3}$ are the radial distortion parameters and $r^{2}=x^{\prime 2}+y^{\prime 2}$ [27].

The tangential distortion which occurs because the lenses are not parallel to the imaging plane. The tangential distortion can be corrected using the following equation.

$$
\begin{aligned}
& x^{\prime \prime}=x^{\prime}+2 p_{1} x^{\prime} y^{\prime}+p_{2}\left(r^{2}+2 x^{\prime 2}\right) \\
& y^{\prime \prime}=y^{\prime}+p_{1}\left(r^{2}+2 y^{\prime 2}\right)+2 p_{2} x^{\prime} y^{\prime}
\end{aligned}
$$

where $p_{1}, p_{2}$ are the tangential distortion coefficients [27]. The above equations are 
employed to calibrate the camera sensors used in this work. Using the two equations 2.8 and 2.9 , it is possible to calibrate the camera for visual odometry work.

\subsubsection{Feature detection and description}

The feature detection step consists in extracting interesting features (or keypoints) on the incoming image. These interesting points are also known as "salient" features as they are points in the image that are interesting such as a corner, peak, etc. Keypoints are divided mainly into corner detectors and blob detectors. Corner detectors attempt to detect an intersection of two lines as a corner in the image while the blob detectors attempts to find a patch in the image that differs significantly from the surrounding area. Generally, blobs are more unique than corners and hence they are more robust to tracking. However, blobs take a considerable longer time to computer in general. There are several corner detection algorithms available in the literature such as FAST (Features from Accelerated Segment Test) [28], Harris [29], Shi-Tomasi [30] among others. The Mars Exploration Rovers use the Harris corner detector. Harris corners computes the differential of the corner score (known as autocorrelation function) to measure changes in image intensity that result from shifting of an image patch. Because of the simplicity and the low computational impact of this corner detector, it is ideal for low computation environments. Another widely used corner extractor is FAST. This feature extractor has been used by several SLAM systems such as [12], [31]. The most widely used blob detectors are SIFT (Scale Invariant Feature Transform) [32], SURF (Speed Up Robust Features) [33]. SIFT and SURF are considerable more computationally intensive than FAST and Harris corner detectors.

There are several new blob detectors such as ORB (Oriented FAST and rotated BRIEF) [34] and Binary Robust Invariant Scalable Keypoints (BRISK) (Binary Robust Invariant Scalable Keypoints) [35]. ORB, as its name indicates, uses the FAST feature detector but it adds a orientation component to the detector. Once the feature is detected, it uses the rotation invariant BRIEF [36] descriptor to describe the feature. The main ORB paper shows how ORB compares to SIFT and SURF and outperforms them in some scenarios while being orders of magnitude more computationally efficient [34]. BRISK is also a new feature descriptor that achieves similar performance to SURF while being an order of magnitude faster. BRISK uses a scalespace keypoint detector which uses FAST features detected in octave layers of the 
image pyramid as well as in layers in-between [35].

One of the main goals of this thesis is to test these new detectors for the purposes of Visual Odometry and Visual SLAM. There are several measures that can be utilized to compare the performance of feature detectors and descriptors in the context of visual odometry algorithms. For the purposes of this thesis, the metrics "stability" and "accuracy" of the feature detection algorithms are used to compare them [37]. In this thesis, the widely used sample image sequence "grafiti" is employed. This image sequence is used by all papers comparing the algorithms [37] [35]. In the case of ORB, the magazine dataset sequence is used as it is quite similar to the graffiti dataset [34]. Another work comparing the algorithms is presented in [38]. A less quantitative comparison of the algorithms is presented in [23] which is used mainly for comparison in this thesis.

In the Table 2.1, several algorithms were compared. A quantitative number from 15 was given to both the stability and accuracy metric. In this case, the higher number represent the higher stability/accuracy. The speed criterion given in milliseconds was tested by the author on a Core i7-2720QM @ 2.2Ghz, 8Gb of RAM. The specific implementation of the algorithms used to run the speed tests were obtained from the Open Source Image processing library OpenCV.

"Stability" refers to the ability to detect the same feature across the viewpoint change. This metric is also known in some papers as the repeatability percentage. This metric is measured in the literature and this thesis by rotating the viewpoint by a certain degree in the $\mathrm{z}$ axis (or yaw) and measuring the number of times that the same feature can be observed and matched in the next image. The work of [37] presented results for 20, 30 and 40 degrees rotation for SURF, SIFT, Censure, Harris, FAST. This thesis employs the measurement of 30 degrees as it is representative of visual odometry cases. The results were presented in percentages in the work of [37]: 100 percent referring to all features matched and 0 percent referring to no features matched. The results on the grafiti sequence were $\mathrm{SIFT}=58, \mathrm{SURF}=57, \mathrm{CENSURE}$ $=58$, Harris $=22$, FAST $=25$. This numbers are used to compute the 5 - 1 scale used in Table $2.1(\mathrm{SIFT}=5, \mathrm{SURF}=5, \mathrm{CENSURE}=5$, Harris $=2, \mathrm{FAST}=2)$. The paper of BRISK [35] includes a table comparing BRISK to SURF on the grafiti sequence. As in the work of [37], the work of [35] introduces a percentage stability number. The percentage for BRISK in this case is 58 percent and for SURF is 70 percent. For this reason, the attached stability number for BRISK is 4 in Table 2.1. In the case of 
ORB, the paper described in [34] presents a repeatability percentage to compare the SURF, SIFT and ORB. The percentage of inliers of SURF for the magazine dataset is 58 percentage while the percentage for ORB is 48 percent. For this reason, the perfomance number of 4 is assigned to ORB.

"Accuracy" refers to the localization error of feature across the viewpoint change [37]. In order to test for the accuracy error, it is possible to analyse the number of frames with small numbers of inliers (good matches) when doing visual odometry. Robust feature detection will produce several good matches while non-robust methods are likely to produce fewer good matches. In the case of visual odometry, 30 matches is the smallest number to properly compute motion. Therefore, accuracy is measured by the number of frames of a certain traverse that had less than 30 good matches after the matching step. The precise visual odometry algorithm used in this tests is explained in more detail in [37]. The results listed in the work of [37] include the number of frames with less than 30 good matches. The results are: Harris $=50$, $\mathrm{FAST}=50, \mathrm{SURF}=9, \mathrm{SIFT}=9, \mathrm{CENSURE}=10$ for the grafiti image sequence. The results included in Table 2.1 can be deduced from the numbers listed above. Similarly, the work of [35] compares SURF, SIFT and BRISK together using the graffiti image sequence. The number of frames for $\mathrm{SURF}=13$ and BRISK=30. Using these numbers, the accuracy value of 3 can be assigned to BRISK in Table 2.1. In the case of ORB, the inlier percentage metric of a single image is the only metric available to compare accuracy to SIFT and SURF [34]. This metric alone cannot be easily translated to number of frames with inlier higher than 30 which is used in this thesis. However, ORB is compared to SIFT and SURF on this publication as well [38]. In the work of [38], the matching ratio is introduced as a measure for accuracy. The dataset employed is the same Freidburg SLAM dataset included by the author in this thesis. The matching ratio is a measure of the retro-projection error of point feature matched in two images. The lower the matching ratio, the better the accuracy of the detector. In the case of the Freidburg SLAM dataset for SURF the matching ratio is 0.3 while for ORB is 0.6. For this reason, the accuracy number of 3 was given to ORB.

The work of [23] also contains a table comparing the different feature detection algorithms and the results listed are very similar to those of Table 2.1. This publication further confirms that the values listed match the expected performance of the algorithms. 


\begin{tabular}{|l|l|l|l|l|}
\hline Detector & Corner/Blob & Time (ms) & Stability(1-5) & Accuracy(1-5) \\
\hline FAST & Corner & 8 & 2 & 1 \\
\hline Harris & Corner & 10 & 2 & 1 \\
\hline SIFT & Blob & 340 & 5 & 5 \\
\hline SURF & Blob & 170 & 5 & 5 \\
\hline CENSURE & Blob & 45 & 5 & 5 \\
\hline ORB & Blob & 23 & 4 & 3 \\
\hline BRISK & Blob & 25 & 4 & 3 \\
\hline
\end{tabular}

Table 2.1: Comparison of feature detector algorithms. Computer system employed is a laptop with Core i7-2720QM @ 2.2Ghz, 8Gb of RAM

Once the features have been detected, an image patch around the feature is converted into a compact form that can be later compared against other features. This step is known as feature extraction. In most cases, extracting merely an image patch around the feature is not a good descriptor as the intensity is likely to change once the viewpoint has changed. One such a method is the sum of square differences (SSD) [39]. This image patch methods will operate only under small movements where changes are not very significant. Another widely used descriptor is the SIFT descriptor. The SIFT descriptor creates a $4 \times 4$ patch and extracts 8 gradients at different orientations. This gradients are normalized to unit length to handle lighting changes and become the 128-element descriptor itself. SIFT descriptor is scale, lighting and rotation invariant which makes it a great candidate for visual odometry. Another robust feature extractor is BRIEF [36] which compares the intensity of image patches around the keypoint and stores the feature as a binary string. ORB utilizes BRIEF but adds rotation invariance which is important in visual odometry cases. The BRISK feature descriptor uses a sampling pattern consisting of points on appropriately scaled concentric circles around the feature [35]. The detector of BRISK is based on FAST, which allows scale and rotation invariance, and a binary descriptor using the pattern described above. Finally, the center-surround feature detectors (CENSURE) features are computed at the extrema of the center-surround filters. The filters are run over multiple scales, using the original image resolution for each scale. The filters are an 
approximation to the scale-space Laplacian of Gaussian and can be computed in real time using integral images. Therefore, CENSURE is very fast and has performance similar to that of SIFT and SURF [37].

Among the above mentioned algorithms, this thesis pays close attention to BRISK and ORB since they are relatively fast while keeping good stability and accuracy. They will be compared against SURF which is generally regarded as one of the most stable and accurate methods.

\subsubsection{Feature matching}

Once the features have been detected and described, the next step is to match the features of the current image to the features of the previous image. There are two main steps in the matching process: match all features with each other in image space and removing false matches.

The first step is to match all the features of the new incoming image to the previous frame. All the feature descriptors of the frames are compared against each other. For the large feature descriptors such as SIFT and SURF, it is time consuming to compare the multidimensional descriptor with each other. Since each feature descriptor has around 128 dimensions and it needs to be compared with several hundred of features, comparing them directly is time consuming (at least linear time search). There are several approaches in the literature to improve the matching by constructing three structures [40] [41]. The most widely used approach when matching large sets of multidimensional descriptors is described in [40]. The work on [40] has also been released as open source software in the FLANN Fast Library for Approximate Nearest Neighbours [42]. This library selects the best algorithm to obtain the approximate nearest neighbours depending on the specific dataset properties [42].

In order to match the smaller binary descriptors used in the case of ORB and BRISK, the normal matching process is applied. The hamming distance is computed between the descriptors. Because the size of the descriptor is smaller, the brute force matching is generally fast.

After the features have been matched in image space, it is possible to pick the top matches to a certain feature. This method for picking the best matches often contains several false matches even with robust descriptors such as SIFT and SURF. For this reason, [32] suggests the "ratio-test" to match features; this algorithm essentially compares a certain feature with its top "n" best matches in image space and computes 
the ratio between the top match and the second top match. If the ratio is less than a configurable number (normally set between 1.5-2.0), then the match is invalid because there are too many similar features to the feature in question. This heuristic value might cause valid matches to be discarded if not adapted to each scene.

There are several other matching algorithms available. Another possibility is to track individual pixels as they move between images (optical flow) [43]. However, this type of tracking algorithms require higher computation than the ratio test. In this work, the "ratio-test" is employed with good results.

\subsubsection{Outlier Rejection}

Once a set of matches is produced, it is possible to estimate the motion between the frames. Despite eliminating bad matches during the matching process, it is highly likely that the data still contains several outliers. Motion blur, noisy images, and other factors can easily cause bad matches even on stable feature descriptors. The most widely used outlier rejection algorithm in Visual odometry algorithms is Random Random Sample Consensus (RANSAC).

\section{RANSAC}

RANSAC is a relatively old and widely used outlier rejection algorithm [44]. The main goal of RANSAC is to compute a model from randomly sampled matches and test this model against the data. The model that produces the highest consensus will be kept and further models discarded. This idea is perfectly suited to visual odometry since there are several matches that might be false and need to be removed. While the number of iterations of RANSAC is high, each iteration is very short since it mainly encompasses comparing a certain model to all matches (several random matches do not even create a proper transform). The number of iterations can be estimated using the Equation 2.10 [44].

$$
N=\frac{\log (1-p)}{\log \left(1-(1-\epsilon)^{s}\right)}
$$

where $s$ is the number of points in the RANSAC algorithm, $\epsilon$ is the percentage of outliers in the datapoints and $p$ is the requested probability of success [44]. This parameters need to be tuned to each specific application. The approach section of this thesis will discuss the parameters in more detail. 
As it can be seen in the equation above, the number of iterations (N) grows exponentially with the number of data points "s" to estimate. For this reason, there are several algorithms proposed that reduce the number of data points. The standard RANSAC algorithm was proposed for un-calibrated cameras and hence it required 5 parameters to estimate. In this thesis, the 3-point RANSAC algorithm is employed. There are publications analysing 2-point RANSAC implementations and even 1-point RANSAC [45].

\subsubsection{Motion Estimation}

After the outliers have been removed and a potential motion estimate is present, it is possible to estimate the motion between the frames. The goal is to estimate a maximum likehood estimate for the camera rotation and translation. One of the first methods noted in [46] solves the estimation problem by modelling the feature uncertainties as ellipsoidal three-dimensional Gaussians and using a covariance-weighted nonlinear least-squares to solve for the position of the robot. This is the main algorithm that is employed in the MER robots [47]. However, most modern visual odometry systems employ some form of bundle adjustment to compute the motion [48] [7] [6]. Bundle adjustment will be discussed in more detail in the following subsection.

\subsubsection{Other front-end approaches}

There are several new approaches at computing relative motion that have achieved good results. The work presented in Large-Scale Direct monocular SLAM [49] uses a direct intensity approach which calculates disparity maps between the incoming frames. Therefore, this method uses all the geometric information of the environment as opposed to just the features as all the approaches described above [49]. This work of [49] employs only a monocular camera with a wide field of view and runs on the CPU without requiring large amounts of computational power. Despite of its benefits, it is a very new method and the author has attempted to use it but obtained poor results due to its need for a highly detailed environment and large field of view camera required. 


\subsection{Back-end system}

There has been several successful Visual SLAM systems in the past including a realtime monocular Extended Kalman Filter (EKF) SLAM system [11]. However, there are some limitations with EKF SLAM such as the limited number of landmarks due to quadratic complexity of the algorithm and potential inconsistencies with the EKF position estimates and the impossibility of re-linearising the cost function after marginalisation [50]. These limitations have motivated work on monocular SLAM to use bundle adjustment as the main underlying position estimator [12].

There are several other probabilistic SLAM techniques such as EKF-SLAM [21], and CEKF SLAM [51], etc. Recently, other authors have proposed unscented versions of SLAM such as Unscented Hybrid-SLAM [52]. Specifically the work of [52] reduces the memory consumption of standard EKF SLAM due to the inherent structure of the unscented Kalman filter. In addition, the work of [52] improves the pose estimation for both features and robot location while reducing time to complete loop closure slightly when compared to EKF-SLAM. The Bayesian-based algorithms described above still have the problem of scaling quadratically with number of features and poses of the robot due to state augmentation [53]. There are several proposed methods to reduce this quadratic time and move it closer to linear time by exploiting sparsification [51] [54], submaps [55], etc. Other problems with Bayesian methods are the data association problem (detecting loop closure, finding previous features, etc) and perceiving the world in a 2D manner (or run in simulation) such as [52] [51]. In the case of visual SLAM, the world can be parametrized in 3D since this information is available.

Another set of algorithms that is present in the literature attempt to estimate only the motion of the robot as opposed to estimating all the features. This family of SLAM algorithms are likely to allow long range robot navigation [53]. Since the motion of the robot and the data association problem can easily be parametrized as a "graph" several Graph SLAM techniques are present in the literature [56]. The most widely used technique to solve optimization problem for graph-based systems is bundle adjustment [57]. For this reason, bundle adjustment has become the state-ofthe-art SLAM framework for visual SLAM [53] [7] [58].

There are several publications that explode bundle adjustment for feature-based SLAM. The work of [12] is designed to operate on small areas and requires a clever choice for "keyframes" which needs to be tuned for specific applications [12]. More 
recent approaches employ relative bundle-adjustment as the main underlying estimation method [7] [6]. However, most work requires the use of computationally expensive feature extraction algorithms such as SIFT [32] and SURF [33] because the accuracy of the feature location is fundamental for the algorithm operation. Another notable proposed algorithm described in [8] employs Features from Accelerated Segment Test (FAST) [59] corner extractor but uses SIFT when the reliability of the FAST estimation degrades.

There is a large family of SLAM algorithms that employ variants of the Iterative Closest Point (ICP) algorithm [60] [61]. While these techniques work in several scenarios, the approach of this thesis is meant to operate also with stereo cameras (where creating accurate point clouds might not be trivial and limited to the field of view of cameras) and hence ICP based techniques are not explored in this thesis.

There is also a large family of work on using RGB-D cameras to achieve visual SLAM. [10] [62] [63] [64] [9]. Most of these proposed algorithms employ assumptions about the RGB-D sensor [10] that might not be valid for the general case of stereo cameras/Flash-LIDAR. In this thesis, there is no assumptions in the measurement model and hence the algorithms should be easily adapted to other sensors.

One of the most widely back-end techniques of feature-based SLAM systems is some variation of Bundle adjustment [65] [8] [58]. The following subsection discuss this technique in more detail.

\subsubsection{Bundle adjustment}

Bundle adjustment is the problem of minimizing the reprojection error between the measured and predicted feature locations, which is expressed as the sum of squares of nonlinear, real-valued function. Therefore, the solution for bundle adjustment is often a nonlinear least-squares problem with a large number of variables. Bundle Adjustment solves for both motion and structure and has been used for several decades as the main Structure for Motion (SFM) algorithm. In the case of visual odometry and back-end systems, this algorithm will solve for the robot positions and the feature positions as well. Recalling Equation 2.1, it is possible to rewrite it as:

$$
x_{j}^{k}=C_{k} \cdot X_{j}
$$


where $X_{j}$ is a visible $3 \mathrm{D}$ point, visible by a camera with $C_{k}$ camera matrix. $x_{j}^{k}$ is the image point of the jth $3 \mathrm{D}$ point seen in the kth camera. The 3D point is generally the centre of an extracted feature using the algorithms described in the sections above. Mathematically, the goal of bundle adjustment is to find the set of camera matrices and $3 \mathrm{D}$ points that minimizes the reprojection error. Assuming Gaussian noise in the measurements, the maximum like-hood solution is:

$$
\arg \min _{X_{j}, C_{k}} \sum_{k, j} d\left(\hat{C}^{k} \cdot \hat{X}^{j}, x_{j}^{k}\right)^{2}
$$

where $d(x, y)$ denotes the euclidean distance between $\mathrm{x}$ and $\mathrm{y}$. This minimization problem contains a large number of parameters (each camera matrix has 11 parameters and each point 3 parameters). In addition, there are several hundreds of features and cameras. Hence, the optimization problem contains generally several thousand parameters. It is possible to re-parametrize the basic bundle adjustment problem as a standard non-linear least squares optimization problem. In order to achieve this, a vector form for all the variables in the problem needs to be introduced. Figure 2.3 [6] illustrates all the variables:

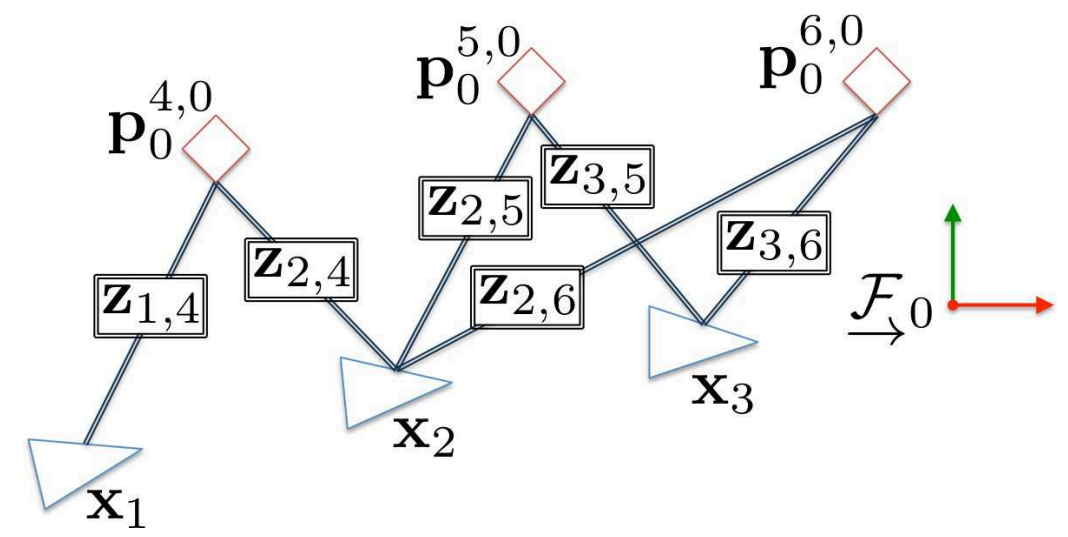

Figure 2.3: Bundle adjustment frame notation.

where $F_{0}$ is the global inertial frame, $F_{k}$ is a frame attached to the robot at time $k, x_{k}$ is the vector representing the position of the robot at time $k, p_{0}^{j, 0}$ is the position of feature $j$ in the global inertial frame and $z_{k, j}$ is the measurement of feature $j$ at time $k$. Using the above definitions, it is possible to define the observation model of a feature $j$ at time $k$ (the measurements are normally distributed):

$$
z_{k, j}=h\left(x_{k}, p_{0}^{j, 0}\right)+n_{k, j}
$$


where

$$
n_{k, j}=N\left(0, R_{k, j}\right)
$$

and $N$ is a normal distribution with zero mean and covariance $R_{k, j}$. It is clear from the equation above that the observation model is specific to $3 \mathrm{D}$ features but not to the sensor used. It is now possible to derive the objective function of bundle adjustment. The derivation comes from the negative log likehood of $p(z \mid x, p)$ [7] [6]:

$$
J=\sum_{k=1}^{K} \sum_{j=1}^{M}\left(z_{k, j}-h\left(x_{k}, p_{0}^{j, 0}\right)\right)^{T} R_{k, j}^{-1}\left(z_{k, j}-h\left(x_{k}, p_{0}^{j, 0}\right)\right)
$$

where $K$ is the number of robot poses and $M$ is the number of features. Minimizing the above objective function yields the maximum like-hood estimate [6]. The objective function can be expressed as a matrix equation by defining the following intermediate vectors (using only 2 features for brevity):

$$
\begin{aligned}
& z=\left(z_{1,1}, \ldots, z_{k, 1}, z_{1,2}, \ldots, z_{K, M}\right) \\
& x=\left(x_{1}, \ldots, x_{K}\right) \\
& p=p_{0}^{1,0}, \ldots, p_{0}^{M, 0} \\
& h(x, p)=\left(h\left(x_{1}, p_{0}^{1,0}\right), \ldots, h\left(x_{K}, p_{0}^{1,0}\right), h\left(x_{1}, p_{0}^{2,0}\right), \ldots,\left(h\left(x_{K}, p_{0}^{M, 0}\right)\right.\right. \\
& R=\left[\begin{array}{cccccc}
R_{11} & & & & & \\
& & & & & \\
& \cdots & & & & \\
& & & & & \\
& & R_{k, 1} & & & \\
& & R_{1,2} & & \\
& & & \ldots & \\
& & & & \\
& & & & R_{K, M}
\end{array}\right]
\end{aligned}
$$


Using the definitions above, the objective function can be re-written as:

$$
J(z \mid x, p)=(z-h(x, p))^{T} R^{-1}(z-h(x, p))
$$

\section{Minimizing the objective function}

There are several techniques to minimize the objective function.

Formally, the minimum of an objective function $\mathbb{R}^{n} \rightarrow \mathbb{R}$ is defined as follows: A function has a local minimum at point $x *$ if $f(x *) \leqslant f(x)$ and $\|x *-x\|<\epsilon$ where $\epsilon$ is a small number and $\epsilon<0$. A point $x *$ is called a global minimum if $f(x *)<f(x)$ for all $\mathrm{x}$.

A necessary but not sufficient condition for a minimum at $x *$ of a differentiable function $\mathrm{f}: \mathbb{R}^{n} \rightarrow \mathbb{R}$ is that the gradient at $x *$ is zero: $f^{\prime}(x *)=0$. This concept can be extended for a multidimensional function (such as the objective function in question) by defining the gradient in Equation 2.21:

$$
\nabla f(x)=\left(\frac{\delta f(x)}{\delta x_{1}}, \ldots, \frac{\delta f(x)}{\delta x_{n}}\right)^{T}
$$

Using the definition in Equation2.21, the point $x *$ might be a minimum of the function if the gradient is equal to zero.

$$
\nabla f(x *)=0
$$

The goal of all non-linear optimization methods is to arrive at $x *$. In order to arrive at $x *$, the parameter vector needs to be optimized iteratively. Defining the parameters as $\beta^{k}$ where $k$ is the iteration number, it is possible to write the optimization of the parameters as:

$$
\beta^{k+1}=\beta^{k}+\Delta \beta
$$

where $\beta^{0}$ is the initial guess and $\Delta \beta$ is the shift vector. The goal of all the different minimization techniques is to find the "best" $\Delta \beta$ that will reach $x *$ quicker. In the case of bundle adjustment(since the parameters of bundle adjustment are the robot pose and feature position): 


$$
\Delta \beta=\left[\begin{array}{l}
\delta x \\
\delta p
\end{array}\right]
$$

The simplest technique to find $\Delta \beta$ is the gradient method. It is defined in Equation2.25:

$$
\Delta \beta=-\lambda_{k} \widehat{J}_{k} F\left(\beta^{k}\right)
$$

where $\lambda_{k}$ is a positive step width that can be adjusted in each iteration. $\widehat{J}_{k}$ is the Jacobian of $\mathrm{F}$ and is defined as follows:

$$
\widehat{J}(\beta)=\left[\begin{array}{ccc}
\frac{\delta f_{1}(\beta)}{\delta \beta_{1}} & \ldots & \frac{\delta f_{1}(\beta)}{\delta \beta_{n}} \\
\ldots & & \ldots \\
\frac{\delta f_{m}(\beta)}{\delta \beta_{1}} & \ldots & \frac{\delta f_{m}(\beta)}{\delta \beta_{n}}
\end{array}\right]
$$

In order to guarantee that this method will reach a minimum $\lambda_{k}$ must be small and hence this method will take a long time to converge.

Another commonly used faster algorithm is the Gauss-Newton method. The Gauss-Newton method uses the Taylor expansion to approximate the objective function. This method converges quadratically towards a local minima. For this reason, it requires a good initial guess, has the potential of diverging on some cases and it clearly is susceptible to local minima [66]. In order to best define this method, the equation 2.20 is revisited. We can define $\bar{x}$ and $\bar{p}$ as the current robot pose and feature position. It is possible to linearise the objective function about its $\bar{x}$ and $\bar{p}$ using Taylor's expansion. The following equation shows the linearisation of the objective function 2.20

$$
J=\left(z-h(x, p)-\left[\begin{array}{ll}
A & B
\end{array}\right]\left[\begin{array}{l}
\delta x \\
\delta p
\end{array}\right]\right)^{T} R^{-1}\left(z-h(\bar{x}, \bar{p})-\left[\begin{array}{ll}
A & B
\end{array}\right]\left[\begin{array}{l}
\delta x \\
\delta p
\end{array}\right]\right)
$$

where:

$$
A=\frac{\delta h}{\delta x}_{\bar{x}, \bar{p}}
$$




$$
B={\frac{\delta h}{\delta p_{\bar{x}, \bar{p}}}}
$$

The $A$ and $B$ are the known as the Jacobians(or $\hat{J}$ as defined before). It is now possible to take the derivative of $\mathrm{J}$ with respect to $(\delta x, \delta p)$ and setting this to zero, it is possible to find:

$$
\left[\begin{array}{l}
A^{T} \\
B^{T}
\end{array}\right] R^{-1}\left[\begin{array}{ll}
A & B
\end{array}\right]\left[\begin{array}{l}
\delta x \\
\delta p
\end{array}\right]=\left[\begin{array}{l}
A^{T} \\
B^{T}
\end{array}\right] R^{-1}(z-h(\bar{x}, \bar{p}))
$$

The above equation can be re-written as:

$$
\left[\begin{array}{cc}
A^{T} R^{-1} A & A^{T} R^{-1} B \\
B^{T} R^{-1} A & B^{T} R^{-1} B
\end{array}\right]\left[\begin{array}{l}
\delta x \\
\delta p
\end{array}\right]=\left[\begin{array}{c}
A^{T} R^{-1}(z-h(\bar{x}, \bar{p})) \\
B^{T} R^{-1}(z-h(\bar{x}, \bar{p}))
\end{array}\right]
$$

It is now possible to rename the above components as:

$$
\left[\begin{array}{cc}
U & W \\
W^{T} & V
\end{array}\right]\left[\begin{array}{l}
\delta x \\
\delta p
\end{array}\right]=\left[\begin{array}{l}
\epsilon_{a} \\
\epsilon_{b}
\end{array}\right]
$$

The system of equations in 2.31 can now be solved for $\delta x$ and $\delta p$. Another method for finding out $\Delta \beta$ is the Levenberg-Marquardt algorithm. Equation 2.30 could be rewritten as:

$$
\left.\left(\widehat{J}_{k}^{T} \widehat{J}_{k}\right) \Delta \beta=-\widehat{J}_{k}^{T} F\left(\beta^{(} k\right)\right)
$$

This is the Gauss Newton method. The Levenberg-Marquardt method combines the Gauss-Newton and the gradient descent method to overcome the divergence problem of Gauss-Newton while keeping good speed. The shift vector $\Delta \beta$ is defined by:

$$
\Delta \beta=-\left(\widehat{J}_{k}^{T} \widehat{J}_{k}+\lambda_{k} I\right)^{-1} \widehat{J}_{k} F\left(\beta^{k}\right)
$$

where $\lambda_{k}$ is a damping factor adjusted in each iteration. If $\lambda_{k}=0$, Equation2.32 becomes the Gauss-Newton method. If $\lambda_{k}=$ inf then Equation2.32 becomes the gradient descent method. For the first iteration, $\lambda_{k}$ is set to a large value to begin the iterations like the gradient descent method. As the solution gets closer to the 
minimum, $\lambda_{k}$ is set to smaller values so it converges quicker to the minimum. The iterations are stopped when a maximum number of iterations is reached or $\Delta \beta$ is below a threshold.

The most time consuming step of both Gauss-Newton and Levenberg-Marquardt is to compute the $\widehat{J}$. The following subsection describe the sparse nature of the Jacobian and how this can be employed to speed up the computation of the Jacobian.

\section{Sparse structure of Bundle adjustment}

Recalling equation 2.31, it is possible to solve for $\delta x$ and $\delta p$. It is clear to see that the naive solution will be expensive due to the large number of features and poses. However, since each equation depends only on one pose and one feature, the Jacobians $A$ and $B$ are very sparse. Figure 2.4 [6] illustrates the Jacobians for a bundle adjustment problem with three cameras and four features.

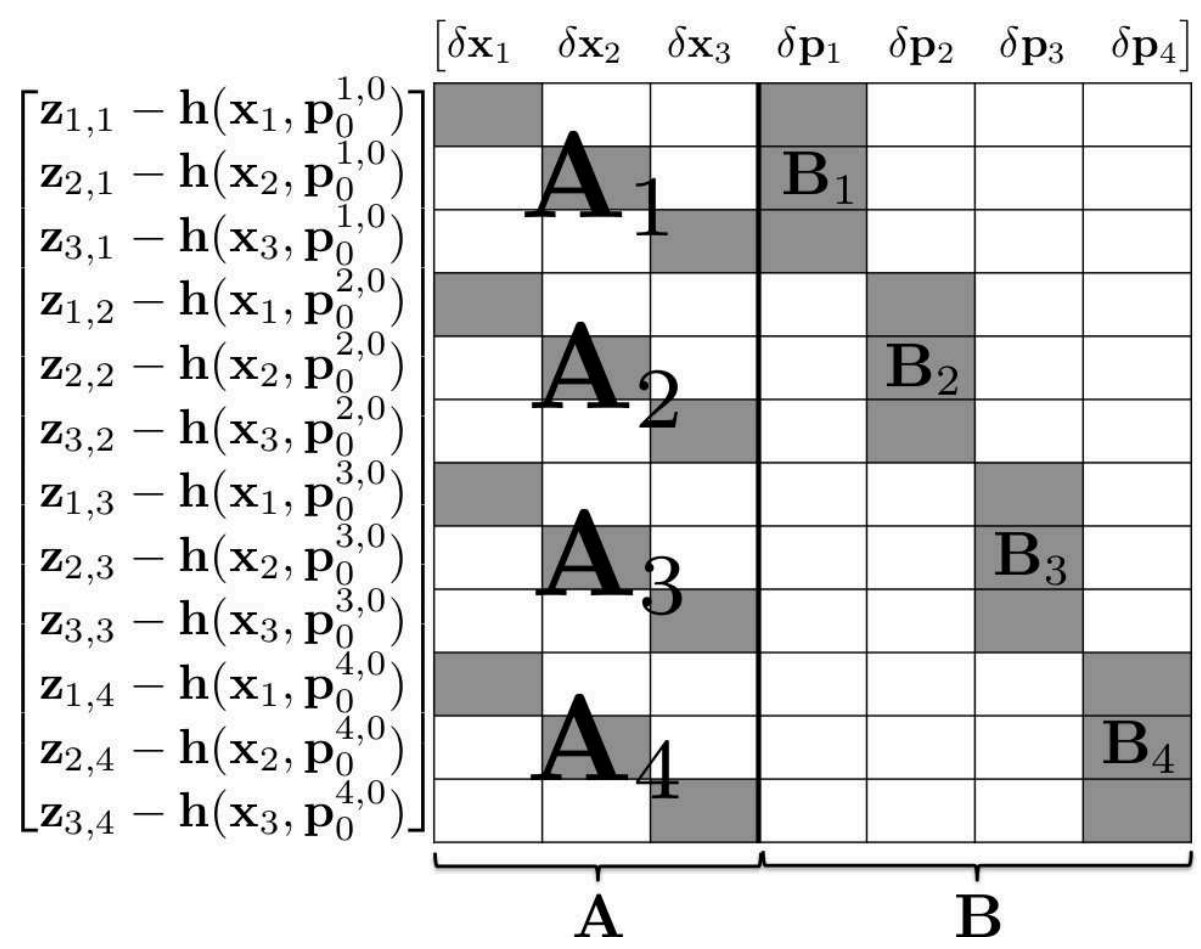

Figure 2.4: Sparse structure of the $A$ and $B$ Jacobians for a bundle adjustment problem of three poses and four features.

Because of the sparsity of $A$ and $B$, it is possible to prove that $U$ and $V$ are both block diagonal [67]. For more information on a theoretical proof of this, please refer 
to [67]. One of the way to solve a spare system is to use the Schur complement. It is possible to pre-multiply Equation 2.31 by the following matrix:

$$
\left[\begin{array}{cc}
1 & -W V^{-1} \\
0 & 1
\end{array}\right]
$$

Using Equation 2.33 on Equation 2.31, one can find:

$$
\left[\begin{array}{cc}
U-W V^{-1} W^{T} & 0 \\
W^{T} & V
\end{array}\right]\left[\begin{array}{l}
\delta x \\
\delta p
\end{array}\right]=\left[\begin{array}{c}
\epsilon_{a}-W V^{-1} \epsilon_{b} \\
\epsilon_{b}
\end{array}\right]
$$

From the equation 2.34, one can note that $\delta x$ solution is now decoupled from $\delta p$. Since $V$ is diagonal, it is not difficult to invert. The matrix $U-W V^{-1} W^{T}$ is dense but is small [67] [65]. Therefore, solving for $\delta x$ is efficient to compute. After $\delta x$ has been found, this can be substituted back to find $\delta p$.

\subsubsection{Graph SLAM}

The goal of this thesis is to provide a back-end system with as little computational load as possible. One SLAM system that employs small computationally load is the graph SLAM approach where the camera poses computed by the visual odometry are stored as nodes on a graph - hence the name graph-SLAM [23]. This approach is used in a several visual SLAM systems [10] [68] [69]. In order to create the graph, each new estimation is added as an edge to the graph and the edge constraints define the following cost function:

$$
\sum_{e_{i j}}\left|C_{i}-T_{e_{i j}} C_{j}\right|^{2}
$$

where $T_{e_{i j}}$ is the transformation between the poses $i$ and $j$ and $C_{j}$ is the camera pose at the position $j$. Therefore, the pose-graph optimization searches for the camera poses that will minimize the above cost function. The rotation part of the transformation makes the cost function non-linear. Because of this non-linearity, it is common to use the Levenberg-Marquardt technique for solving [10]. 


\section{Chapter 3}

\section{Approach}

\subsection{Proposed Approach}

This section will describe in the detail all the components of the proposed VSLAM system.

\subsubsection{System Overview}

Figure 3.1 provides an overview of the VSLAM system.

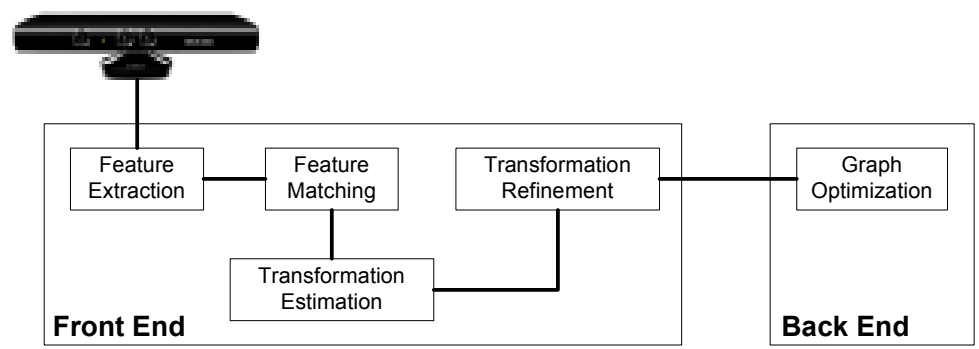

Figure 3.1: System overview diagram

As described in the background section, most visual SLAM systems can be divided into a front end and back end. The front end section estimates the relative motion between the current frame and previous frames. This relative estimation can be aided by the use of a wheel odometry and IMU. However, in this thesis, the Microsoft kinect sensor is the only sensor employed (no IMU or odometry). The back end system employs the estimated relative transformations and their associated uncertainties to build a pose-graph of the robot's traversal. Using the pose-graph, a maximum 
likehood optimization can be used to determine the most accurate traversal of the robot. This optimization will also help to achieve loop-closure in the event that the robot returns to a previously visited area.

\subsubsection{Thesis requirements and implementation overview}

The main goal of this thesis is to come up with an end-to-end visual SLAM solution that can be utilized to compare different algorithms. The main design objectives are:

1. The algorithms used in this thesis must make the overall algorithm was lightweight as possible since its designed to operate in a computationally constrained system

2. The algorithm used must allow for modularity so that each section of the algorithm can be evaluated differently.

3. The algorithm must provide an end-to-end solution and estimate robot motion using the sensor provide.

Due to the requirements listed above, open source algorithms were employed whenever possible to complete the thesis work in time. The following list explains the algorithms used at each step"

- Feature detection: The SURF, BRISK and ORB algorithms used were obtained from the OpenCV library [27].

- Feature description: The SURF, BRISK and ORB feature description algorithms were obtained from the OpenCV library [27].

- Feature matching: The FLANN and Brute force (hamming distance) matching algorithm were obtained from the OpenCV library [27].

- Outlier Rejection: The author was unable to find an implementation of RANSAC that was suitable for this work. For this reason, the author fully implemented a version of 3 point RANSAC. More details will be described in the outlier rejection section of this section.

- Two-frame bundle adjustment: The LibG2O library was employed to implement two frame bundle adjustment. A significant amount of time was spent on 
understanding how to employ LibG2O for the purposes of bundle adjustment and adding requirements between nodes and features.

- Graph-SLAM back-end: The LibG2O library was employed to generate the graph and updated as needed. The implementation side of this thesis concentrated on how to best add nodes to the tree and interfacing with libG2O library.

An important emphasis of this thesis was on the implementation section of the work. A large amount of effort was placed on writing the software on a modular and clean fashion in order to allow other algorithms such as feature detection, matching, outlier rejection or local optimization algorithms to be tested with the overall system. This modularity allows to characterize different algorithms for each of the processing steps.

Another important challenge faced in the implementation section of the thesis was interfacing with sensors and interfacing of the different algorithms. Significant time of the implementation was spent on understanding on how to best operate the Microsoft Kinect sensor. As it will be explained in section 3.1.3, the kinect sensor required synchronization between incoming frames, shadowing error checking and averaging of depth values to be utilized in this thesis. In addition, the different opensource libraries each have their own unique Application Programming Interface (API) and assumptions about data variables. For example, the RANSAC algorithm coded uses RMSE as the measurement of error and LibG2O uses the information matrix to estimate error. A considerable amount of effort was involved in understanding their API and ensuring that the proper data was provided to the algorithms.

The specific details of algorithms used will be described in the next subsections.

\subsubsection{Kinect sensor calibration}

The calibration of a standard pinhole camera was explained in the background section. An in-depth review of the calibration and sensor models of the Kinect sensor can be found in [70]. The Kinect sensor is essentially a infra-red laser emitter, an infra-red camera and a RGB camera. 


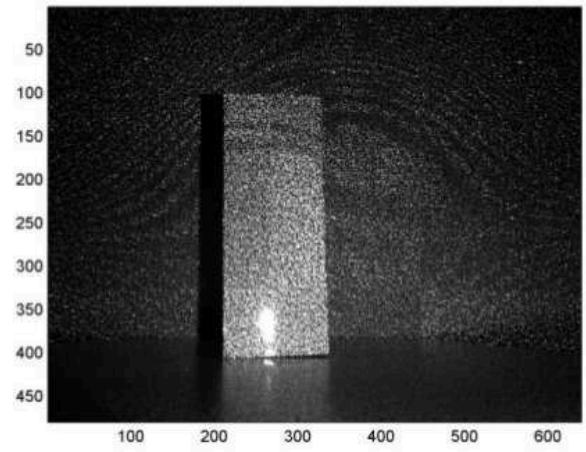

(a)

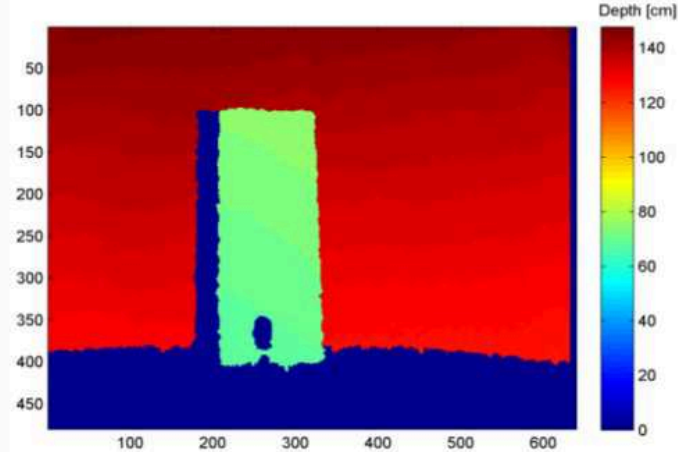

(b)

Figure 3.2: Kinect sensor infrared depth sensing. a) Infrared image of the pattern of speckles projected on a sample scene. b) The resulting depth image

The depth measurements use the triangulation method between the infra-red emitter and the infra-red camera. The emitter projects a single beam with a diffraction grating which produces a constant pattern of "speckles" on the scene. The distance to a certain object can be reconstructed from the shift in the speckle pattern [70].

The Kinect sensor provides a 640x480 disparity image as it can be seen in figure 3.2 [70]. In order to express the 3D coordinates of a certain point in the space, a coordinate system at the base of the infra-red receiver needs to be defined. The $\mathrm{Z}$ axis of this axis is orthogonal to the image plane facing towards the scene. The $\mathrm{X}$ axis is perpendicular to the $\mathrm{X}$ axis in the direction of the baseline. Figure 3.3 [70] illustrates the axis:

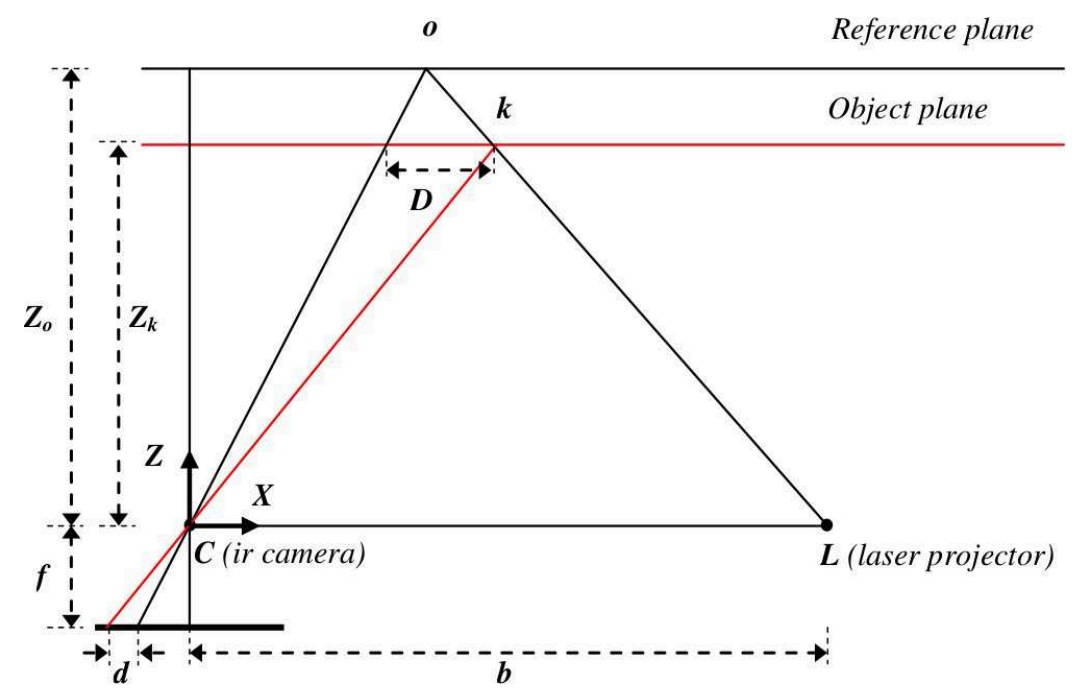

Figure 3.3: Illustration of the Microsoft kinect optical axis and how depth is measured. 
where $\mathrm{b}$ is the baseline, $Z_{o}$ is the distance to the reference place speckle and $Z_{k}$ is the plane of the object being measured.

The Kinect sensor is modelled as a infrared camera without tangential lens distortion. From Figure 3.3, one can write:

$$
\frac{d}{f}=\frac{D}{Z_{k}}
$$

where $f$ is the focal length of the infra-red camera and $D$ is the displacement of the point k in object space. From similarity of triangles in Figure 3.3 one can obtain $D$

$$
\frac{D}{b}=\frac{Z_{o}-Z_{k}}{Z_{o}}
$$

Therefore, $Z_{k}$ can be expressed as:

$$
Z_{k}=\frac{Z_{o}}{1+\frac{Z_{o}}{f b} d}
$$

Similarly, it is possible to define the $\mathrm{X}, \mathrm{Y}$ coordinate of the point.

$$
\begin{aligned}
& X_{k}=-\frac{Z_{k}}{f}\left(x_{k}-c_{x}+\delta x\right) \\
& Y_{k}=-\frac{Z_{k}}{f}\left(y_{k}-c_{y}+\delta y\right)
\end{aligned}
$$

where $x_{k}$ and $y_{k}$ are the image coordinates of the point, $c_{x}, c_{y}$ are the coordinates of the principal point and $\delta x, \delta y$ are corrections for lens distortion. The radial distortion model described in the background section is used to calibrate $\delta x, \delta y$ in this thesis. Using the equations above, it is possible to compute the object $(x, y$, Depth) from the original depth image. [70].

The calibration for the RGB camera on the kinect was carried out using the equations described in the background section and the OpenCV camera calibration tools. The widely used OpenCV calibrations tools were also employed to calibrate the Kinect sensor. [71].

It is also necessary to model the noise on the measurements in order to create a covariance matrix of the feature position (this is used for the bundle adjustment step $R$ matrix). The Kinect can have three main error sources: the measurement set-up, the internal sensor error and the object surface [18]. Modelling the object surface is a 
difficult task since the environment cannot be pre-mapped and hence this error source is ignored. The measurement set-up error refers to error in lighting and geometry of the surface being measured. Since the environment is likely to have complex geometry and changes in illumination, this type of error is also ignored. Therefore, the only error that is modelled is a random error on the measurement. Once the calibration parameters are accurately determined, one can define $d^{\prime}$ is a random variable with normal distribution representing the disparity. The variance of $d^{\prime}$ is $\sigma_{d^{\prime}}^{2}$. By applying this variance of disparity, one can obtain the variance of the measurement as : [70].

$$
\sigma_{z}=\left(\frac{m}{f b}\right) Z^{2} \sigma_{d^{\prime}}
$$

where $\sigma d^{\prime}$ and $\sigma Z$ are the standard deviation of the measured disparity and the standard deviation of the depth. Equation 3.6 clearly shows how the random error of the depth is proportional to the square distance from the sensor to the object. Since depth is also involved in calculation the object coordinates, the error in determining $\mathrm{X}$ and $\mathrm{Y}$ also has the same second order growth with respect to the distance.

$$
\begin{gathered}
\sigma_{x}=\left(\frac{m x}{f^{2} b}\right) Z^{2} \sigma_{d^{\prime}} \\
\sigma_{y}=\left(\frac{m y}{f^{2} b}\right) Z^{2} \sigma_{d^{\prime}}
\end{gathered}
$$

Using the variances above, one can build the covariance matrix of estimated position of a certain feature. Because there is no strong correlation between the $\mathrm{X}, \mathrm{Y}$ and $\mathrm{Z}$ variables, the covariance matrix for each feature is equal to:

$$
R_{\text {feature }}=\left(\begin{array}{ccc}
\sigma_{x}^{2} & 0 & 0 \\
0 & \sigma_{y}^{2} & 0 \\
0 & 0 & \sigma_{z}^{2}
\end{array}\right)
$$

\section{Shadowing error}

Another important issue with the Kinect sensor that requires consideration is the shadowing error due to the baseline between the infra-red emitter and infra-red camera. An example of this shadow effect can be seen in Figure 3.4 [18]. 


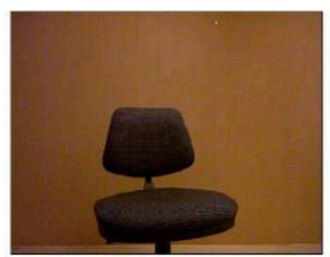

a)

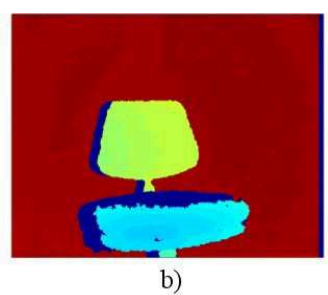

b)

Figure 3.4: a) RGB image of a chair b) Depth image of the same chair. A shadow image on the left of the chair (depth equal to 0 ). This occurs due to the relative position of the infra-red emitter and the infra-red camera.

As it can be seen in Figure 3.4, the chair has a shadow to the left side. This can cause serious estimation problems since the depth of any feature present on that side will give an erroneous measurement. In addition, it is possible to prove that the variance on the depth also changes rapidly near edges [18]. For the reasons above, special care needs to be taken with features that appear near edges.

If the depth information of a certain feature is not present, the descriptor is discarded. For all other descriptors, the average of the depth in the descriptor's size area is employed. This ensures that the depth is representative of the position of the feature in actual space and makes the depth value used more resistant to potential noise in the measurement.

\subsubsection{Front End System}

Once the Kinect camera has been calibrated, it is possible to use the incoming data for motion estimation. The front end system has been designed around the standard visual odometry pipeline from stereo systems [22]. The goal of this algorithm is to incrementally estimate the relative motion between incoming frames. Two camera positions at adjacent time instants $k-1$ and $k$ are related by the rigid body transformation $T_{k, k-1} \epsilon \Re^{4 x 4}$ of the following form:

$$
T_{k, k-1}=\left[\begin{array}{cc}
R_{k, k-1} & t_{k, k-1} \\
0 & 1
\end{array}\right]
$$

where $R_{k, k-1} \epsilon S O(3)$ is the rotation matrix and $t_{k, k-1} \epsilon \Re^{3 x 1}$ is the translation vector. Therefore, the main task of the front end will be to compute the relative transform $T_{k, k 1}$ from the images $I_{k}$ and $I_{k-1}$. 
One of the most computationally expensive steps in visual odometry is the feature detection and feature descriptor extraction [8]. The goal in this thesis was to employ a computationally inexpensive feature detector and extractor while keeping the estimation accuracy similar to that of a system employing expensive feature detection/extraction.

\section{Feature Detection/description}

Several feature detection and extraction algorithms were tested. Run-time and estimation accuracy were used to evaluate different algorithms. Since SURF is proven to provide similar results to SIFT [33], this work used SURF as the benchmark for accuracy. This work also evaluated BRISK [35] and Oriented FAST and Rotated BRIEF (ORB) [34]. While FAST has been employed in several visual SLAM systems, [12], [8], the ORB feature detector was preferred as it improves on the features of FAST to add rotation invariance [34]. The feature descriptor algorithm used were the same algorithm as the feature detection.

\section{Feature matching}

As part of the front end, each new incoming frame is matched to the previous frame. In order to ensure that enough matches are available for the transformation estimation step, the matching process for the BRISK and ORB algorithms is adaptive. One can define each pose of the robot as $X_{n}$; the current frame of the robot as $X_{i}$ and the previous frame as $X_{j}$, the landmarks observed on each frame as $Y_{n}$ and their respective descriptors as $D_{n}$. Each descriptor in $X_{i}$ frame is compared to all the descriptors in the $X_{j}$ frame. For the SURF algorithm, the fast matching of binary features algorithm [72] implemented in the Fast Library for Approximate Nearest Neighbours (FLANN) is used. For the BRISK and ORB feature descriptors, a brute force matching algorithm using the hamming distance is used (since these algorithms have a binary feature descriptor). Only the $K$ top matches of this process are stored. After this step is completed, the ratio between the first and second top matches is obtained as described in [32]. If the ratio is higher than a tunable parameter, the match is stored and later employed for transformation estimation. For the SURF detector, this ratio matching process produces enough matches for transformation estimation (minimum of 10 matches).

However, for the BRISK and ORB algorithms, a extra adaptive step is required to 
produce enough good matches. A minimum-distance-multiplier is introduced. Firstly, the global minimum distance between the keypoints is found. A potential match is then considered as "good" if the ratio between the first and second matches is larger than the tunable ratio parameter or if the image-space distance is less than the minimum-distance-multiplier multiplied by the minimum global distance. The comparison process is then repeated by increasing the minimum-distance-multiplier in every run until enough matches are obtained. This extra adaptive step greatly improves the ability for BRISK and ORB to generate enough matches for the transformation estimation step. The following algorithm describes the procedure explained above.

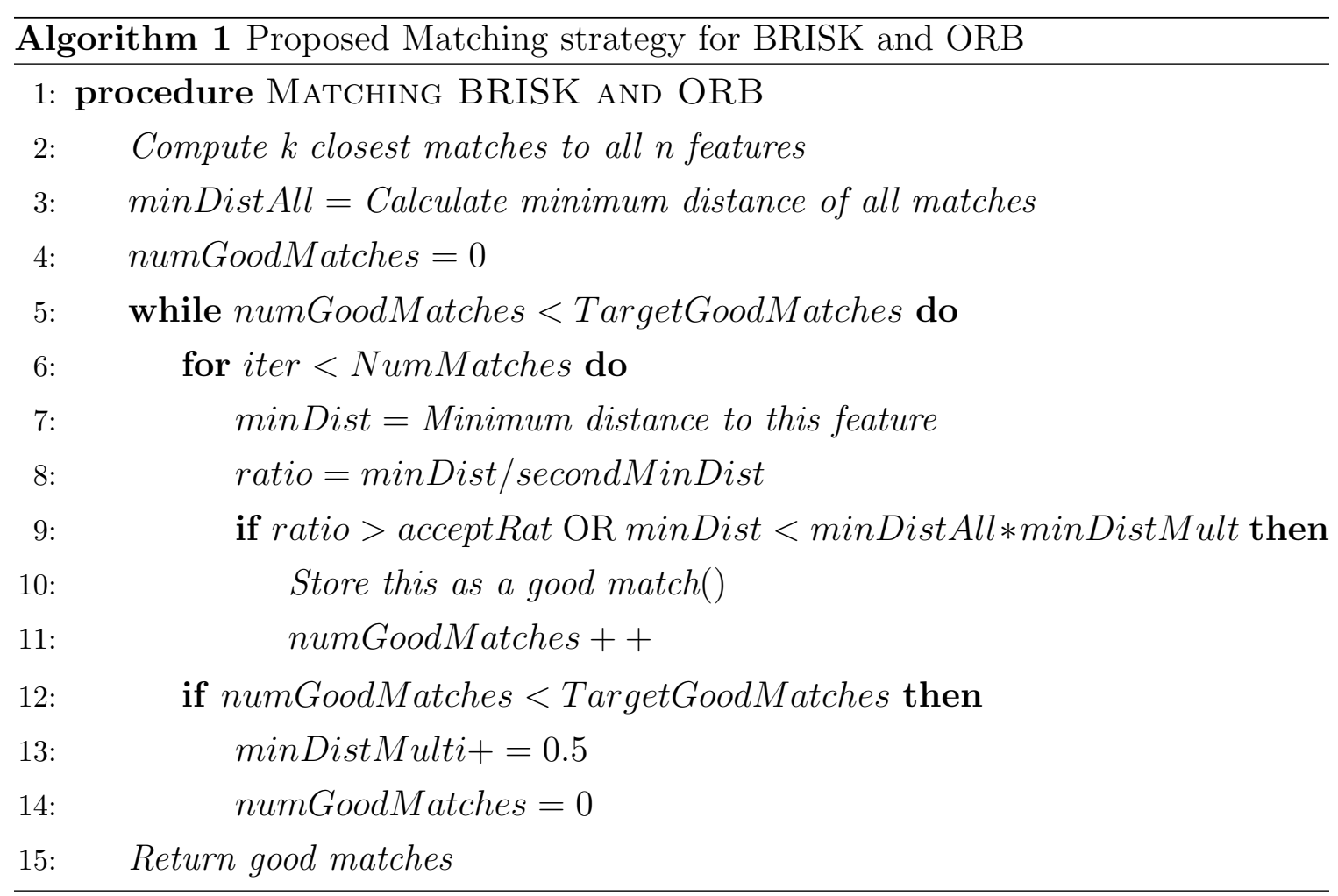

The minDistMulti parameter of 2.0 and the increment of 0.5 were determined heuristically after several tests. These parameters should be adapted according the scenes.

\section{Outlier Rejection}

The matching process produces several matches that are not always correct. In order to reject outliers and estimate the rigid transformation between the consecutive 
frames the widely used 3-point RANSAC algorithm is used [44] [23]. Firstly, 3 random matches are picked to produce a potential transformation. If the potential transformation is valid, the root mean square error (RMSE) of the euclidean distance between the transformation and all the matches is calculated. If the RMSE of a certain match is above a tunable threshold, the match is considered an "outlier" and removed from the list of matches. The average of RMSE values for all inlier matches is then compared against the average RMSE of the "best" transform so far. This standard RANSAC algorithm described above achieved good estimation results in when used with SURF matches. It was observed however that just using the average RMSE as a measure of the accuracy of the transform does not yield good results when using BRISK and ORB matches. Since the BRISK and ORB detectors will inherently produce less matches with higher outliers, some false transformations created from outliers will yield better average RMSE since the number of inliers was very low. For this reason, the evaluation of the best transform in this thesis takes into consideration both the average RMSE and the number of inliers as well. The idea is that the "best" transform should be the one with the lowest number of inliers and the smallest average RMSE. A $\Delta$ Inlier variable was introduced in order to keep number of inliers in consideration when comparing against the "best" transform so far. The final equations used are given by:

$$
\begin{gathered}
\Delta \text { Inlier }=\frac{\text { Inlier Num }_{\text {best }}}{\text { Inlier Num }_{\text {curr }}} \\
R M S E_{\text {curr }}=R M S E_{\text {curr }} * \Delta \text { Inlier }
\end{gathered}
$$

The introduction of the $\Delta$ Inlier helped to reduce the number of false transformations with low inlier count and low average RMSE. The above RANSAC procedure is summarized in a algorithm form: 


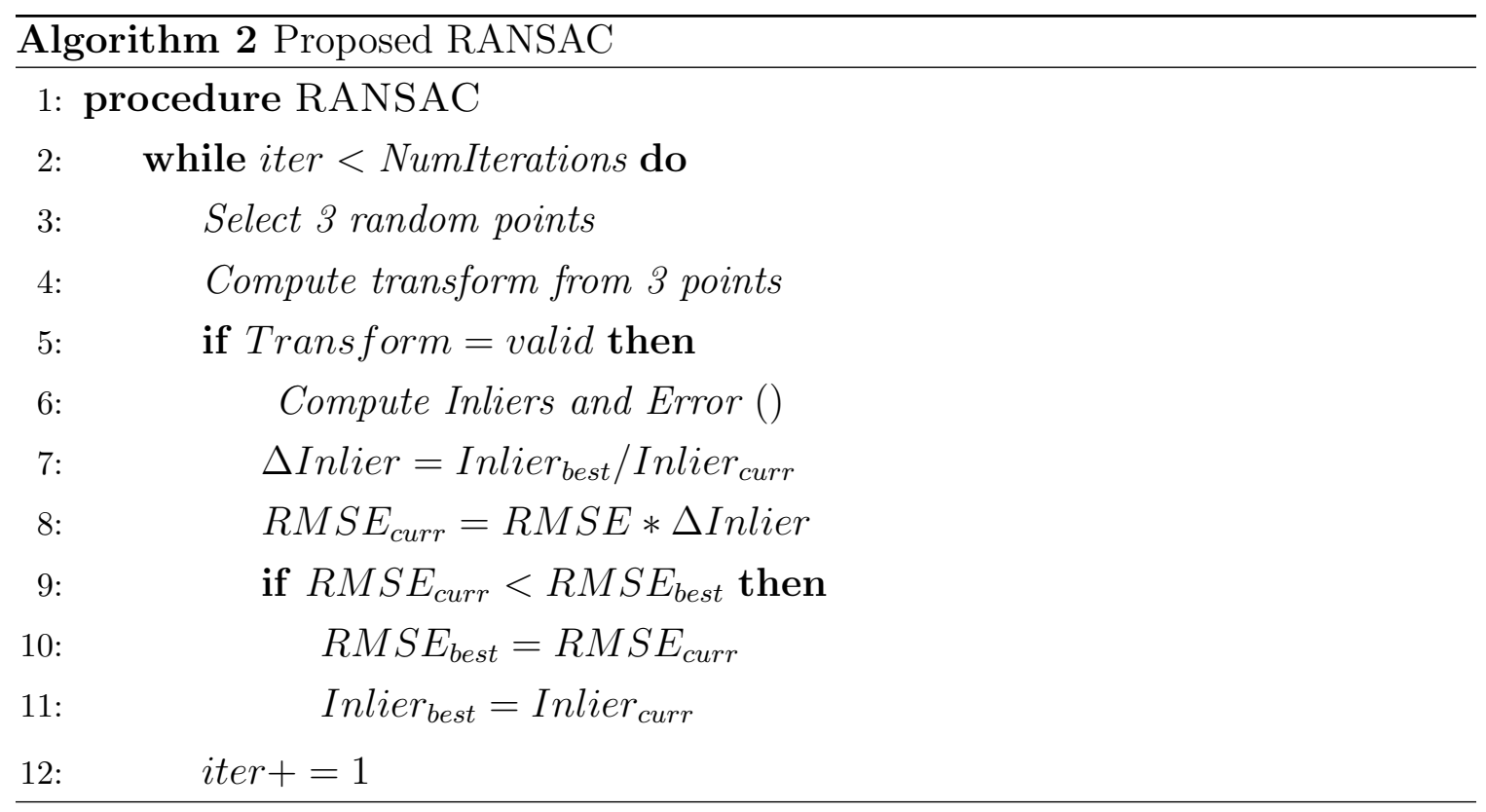

Another important consideration of the RANSAC algorithm is the maximum number of iterations that it must run. The standard equation employed (as outlined in the background section) is:

$$
N=\frac{\log (1-p)}{\log \left(1-(1-\epsilon)^{s}\right)}
$$

Using a $p=0.99, s=3$ and $\epsilon=0.5$, the total number of iterations is 35 . It is recommended in practice to add a safety factor of 5-10 [23]. In this thesis, a safety factor of 6 was employed. The total number of iterations is 210. (it is important to note that this does have a large effect on performance since RANSAC is fast).

Once the initial transformation has been estimated, it is recommended in the literature to optimize the estimation further [22] in order to minimize the error introduce by each consecutive estimation.

\section{Transformation refinement}

Despite the efforts to reject outliers, it is recommended to minimize the estimation error in each consecutive estimation. The transformation estimation step provides a good initial guess and further optimization can be carried out to improve the transformation estimation. In this thesis, we use the approach followed by [62] dubbed "Two-frame bundle Adjustment". Once the RANSAC step is completed, a small 
bundle adjustment problem is setup between the current frame, the past frame and the inlier features. In this thesis, the two-frame bundle adjustment optimization is carried out using the Gauss-Newton approximation since a good initial estimate is already present. Since the optimization in question only includes the 2 camera poses and the inlier features, the Jacobian will exhibit a highly sparse block structure. This sparsity is exploded to reduce computational load [57]. For more details regarding sparse bundle adjustment, please read the background section.

The computational complexity of sparse bundle adjustment is $O\left(m^{3}+m n\right)$ where $\mathrm{m}$ is the number of camera poses and $\mathrm{n}$ is the number of features [73]. In the case of two-frame sparse bundle adjustment the fact there is only 2 camera poses greatly reduces the computational complexity. The precise number changes according to the number of matches between each camera pose. When adding the edges between the camera poses or between camera poses and features position, it is required to add the covariance matrix on the transformation. The libg $g^{2}$ library uses the information matrix which is the inverse of the covariance matrix. The steps followed to achieve the two-frame bundle adjust are:

1. Set the previous frame as a fixed frame on the two-frame graph

2. Create a new node in the graph and add the transformation as the edge constraint and add its covariance matrix

3. For each feature on the previous frame, add the transformation as an edge between the previous frame and each feature. In addition, add the measurement covariance matrix for each transform.

4. For each feature on the current frame, add the transformation as an edge between the current frame and each feature.

5. Run the Gauss Newton optimization to minimize the retro-projection error The covariance matrix employed between the nodes and the features is the same as explained in Equation 3.9.

In order to implement this optimization, the widely used $g^{2} o$ library was used [65]. As mentioned earlier, the Gauss-Newton method was employed since a good start guess is present. The linear solver CHOLMOD was used to solve the system of equations. There is no need to add the information matrix of the transformation since it is provided as an estimate and used as an initial step in the optimization. 


\subsubsection{Graph SLAM (back end)}

Because of the computational requirements of this application, it is important to keep the computation of the pose graph and its related optimization workload low. In order to do this, only the pose of the robot along with its associated covariance are kept on the pose graph. The 3D locations of the features are discarded unlike in the two-frame sparse bundle adjustment optimization. As described in the background section, the edges of the pose-graph have the following cost function:

$$
\sum_{e_{i j}}\left|C_{i}-T_{e_{i j}} C_{j}\right|^{2}
$$

where $T_{e_{i j}}$ is the transformation between the poses $i$ and $j$ and $C_{j}$ is the camera pose at the position $j$. Therefore, the pose-graph optimization searches the camera poses that will minimize the above cost function. The rotation part of the transformation makes the cost function non-linear. In this thesis, the non-linear optimization algorithm used to solve the pose-graph optimization is Levenberg-Marquardt. The computational complexity of this graph optimization is $O\left(\mathrm{~m}^{3}\right)$ when $\mathrm{m}$ is the number of camera poses. This is faster than the standard bundle adjustment complexity which equals to: $O\left((m+n)^{3}\right)$ or the sparse bundle adjustment $O\left(m^{3}+m n\right)$ [73]. The implementation employed the library $g^{2} o$ which supports Levenberg-Marquardt optimization [65]. The optimization was run until it reached a tunable parameter $c h i_{2}$ of 0.01 was reached. The linear solver CHOLMOD was employed [65].

An important question is which of the incoming frames (coming at around $30 \mathrm{~Hz}$ from the sensor) should be added as node on the graph. If too many nodes are added, the pose graph optimization will take a long time to complete. If too few nodes are added, the optimization will not have enough information to properly optimize the traverse and hence it will not correct any present errors. This problem is a very active area of research in graph SLAM at the moment [74]. In this thesis, a two step process is present. The first step include skipping the current frame if less than 10 frames have passed between the current frame and the last frame that was added to the tree as a node. Since each frame contains its "Frame ID", it is simple to check the difference between the "Frame ID" to determine whether 10 frames has passed. The second step includes a simple tunable distance parameter. For each new incoming frame with a successful transformation out of the front-end system, the euclidean 
distance to the previous node in the graph is computed. If this distance is larger than a tunable parameter $(0.1 \mathrm{~m}$ in this case $)$, the current frame is added as a node in the tree.

Another important consideration when building the pose-graph is the choice of the previous nodes. If a new incoming $i$ node is linked against every single node in the pose graph, the processing time will be greatly increased. In this thesis, a configurable number of $n$ edges are created for each new $i$ frame (or node). The challenge is to locate the $n$ closest nodes that best represent the location of the new $i$ node. The directly previous nodes (i.e. $i-1, i-2 \ldots$ ) certainly provide a good match for closest nodes to the new $i$ node but they will not provide loop closure capabilities. In this thesis, the $m$ closest nodes have been chosen. $m$ is a configurable parameter set to 2 or 3 depending on the environment. In this case, $m$ must be less than $n$. Once the previous nodes have been added, it is necessary to try and locate which other nodes are closest to the node in question. This step is generally known in the SLAM literature as loop closure. There are several systems available in the literature for loop closure detection. Most systems employ image recognition and appearance based SLAM [75] [76]. However, these appearance-based SLAM systems are used for very large navigation problems (several hundreds of kilometres).

In this thesis, a simpler graph-based approach is used. The goal is to detect loop closure while keeping the processing as small as possible. A simple approach is to look on the main graph for the closest nodes to the current node in question. This approached was employed by [10]. This approach searches for $k$ nodes in the geodesic (graph-)neighbourhood of the previous nodes. In order to find these nodes, the minimal spanning tree of limited depth (tunable parameter, set to 3 in this case) from the pose graph, with the sequential predecessor as root node is computed. The $m$ previous nodes already selected are removed to avoid repetition. After this, only $k-m$ nodes are drawn randomly from the list of nodes in the minimum spamming tree. This approach is capable of locating small loop closures as well as linking the new node to further previous nodes. The parameters $m$ and $k$ can be tuned depending on the experiment. In this thesis, $m=2$ previous nodes are used and $k=3$ geodesic nodes are used. This accounts to a total of $n=5$ edges for each new incoming node. In order to estimate the transformation between the 5 nodes chosen to the current node, the RANSAC algorithm described above is used. It is important to note that the two-frame bundle adjustment step is not used since the initial transformation 
provided by RANSAC is good enough for the pose-graph.

In order to create edges, it is required that the information matrix of the new edge (or transformation in this case) is present. In order to compute the information matrix, a identity matrix multiplied by the $R M S E^{2}$ of the transformation was used. Since the information matrix is essentially the inverse of the covariance of the measurement, the covariance matrix was inverted to obtain the information matrix. In order to summarize, the steps followed to add a new edge to the graph are listed below.

1. Receive the latest frame. If the transformation to the last frame is invalid, skip the current frame

2. Check the current frame ID. If the current "Frame ID" is less than 10 frames after the latest node "Frame ID", skip the current frame.

3. Compute the distance of the current frame to the latest added node in the graph. If the euclidean distance is less than 0.25 , skip the current frame.

4. Pick the $m$ previous nodes to the current node.

5. Create the minimum spamming tree from the graph with depth of 3 with the $i-1$ node as head. This will produce $p$ nodes.

6. Remove $m$ nodes from the $p$ nodes

7. Pick $k$ nodes from the $p-m$ nodes at random. Create a list of nodes $n$ where $n=k+m$

8. Match the current node with the $n$ nodes. Run RANSAC to reject outliers and estimate motion.

9. Add only the nodes that RANSAC successfully estimated a transformation.

10. Create the information matrix for the current transformation. In order to compute the information matrix, the covariance matrix is required. To obtain the covariance matrix, a identity matrix multiplied by the $R M S E^{2}$ from RANSAC of the transformation was used:

$$
R_{\text {trans }}=R M S E^{2} * I_{6}
$$


The parametrization of the $\mathrm{Se}(3)$ transformation between the edges in $\operatorname{lib} g^{2} o$ uses the (x,y,z,roll,pitch,yaw) parametrization and hence the dimension of the covariance matrix. In order to obtain the information matrix, it is possible to simply invert the covariance matrix.

11. Run the graph optimization

The optimization system uses the first node as the global reference frame. The minimum spamming tree helps locating nodes that might provide loop closure and the depth on the spamming tree prevents the system from picking nodes that will not be in the vicinity of the newly added node.

There are several systems employing a relative optimization system in order to reduce the optimization time [7] [6]. In the work of [7], the global initial node approach is used. Relative bundle adjustment is a complex algorithm and there is no opensource implementation of this algorithm publicly available at the time of this thesis. 


\section{Chapter 4}

\section{Experiments}

\subsection{Experiments}

This section discusses the experimental results. The first area used to test is an indoor area with an office environment dubbed "Carleton indoor" dataset. Figure 4.1 illustrates the tested environment.

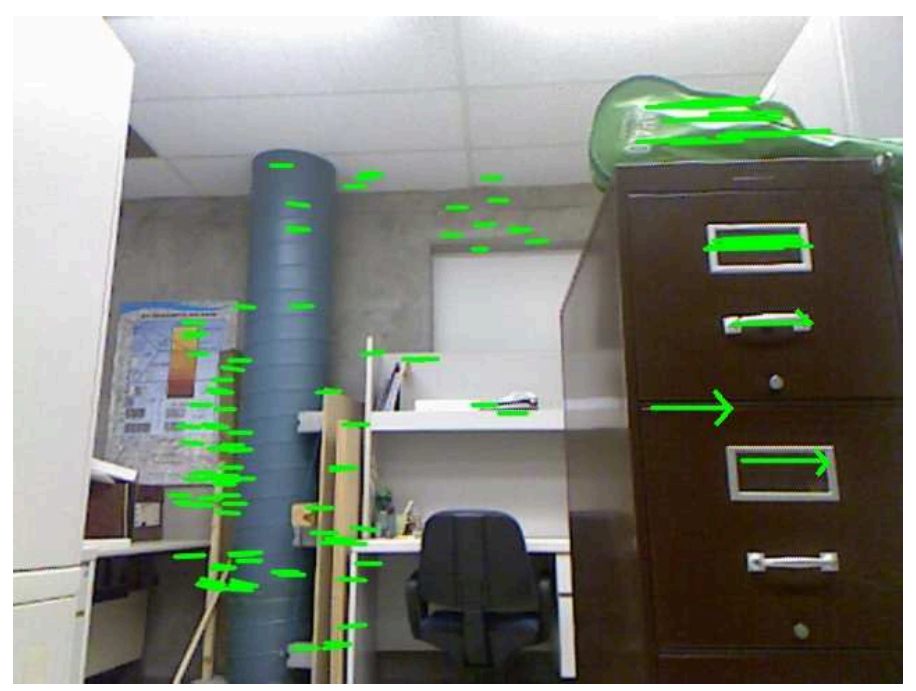

Figure 4.1: Sample image from the office testing environment. The green arrows represent the optical flow generated with the final matches once RANSAC has removed outliers. (the feature detection/descriptor algorithm used was SURF)

In Figure 4.1, the feature flow be observed. Three features on the right side have arrows displaying the direction of the feature flow. The feature flow refers to the visualization of how a feature has moved between frames as analysed by the frontend algorithm. The feature in question existed in a previous frame. The position of 
the feature in the previous frame is depicted by the tail of the arrow. The position of the feature in the current frame is depicted by the end of the arrow. The rest of features do not have a arrow head in order to preserve space.

A set of 30 images were captured sequentially to test the performance of the algorithm. The images contain translations in all angles as well as rotations of up to 30 degrees. Figure 4.2 illustrates the viewpoints of the images captured and their order.

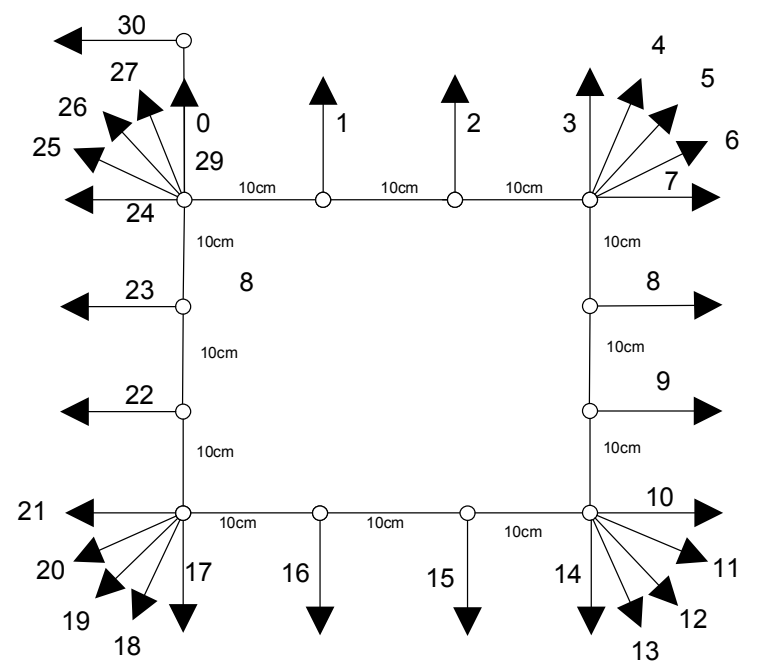

Figure 4.2: Carleton Indoor Dataset overview. The viewpoints are illustrated by arrows and the sequence by numbers

It is important to note that this dataset was specifically gathered to test the ability of the SLAM system for estimating rotation (each 22.5 degrees). A large rotation such as 22.5 degrees causes almost $1 / 3$ of the original image to be no longer available in the next frame. The author attempted to create a dataset with 45 deg rotation (where almost $2 / 3$ of the image was no longer available in the next frame) and was unable to recover a transform even when using SURF features. In addition, frame 30 provides a test for the "kidnapped" case where the previous frames do not have any relation to the next frame. In this case, Frame 24 is the only frame that will provide a valid transform to frame 30. To summarize, the Carleton dataset tests for the following features of the algorithm:

1. Translation of $10 \mathrm{~cm}$

2. Rotation of 22.5 degrees (2/3 of the frame available for next image) 
3. Kidnapped robot case

\section{Loop closure detection}

The second area used for testing is a publicly available dataset described in [77]. The dataset used is the "Freidburg-SLAM" dataset which was carried out using a Pioneer P3DX Robot and a Microsoft Kinect. The calibration values for the Kinect sensor were provided by [77]. This dataset also includes highly accurate ground-truth data from a high-speed indoor localization system [77].

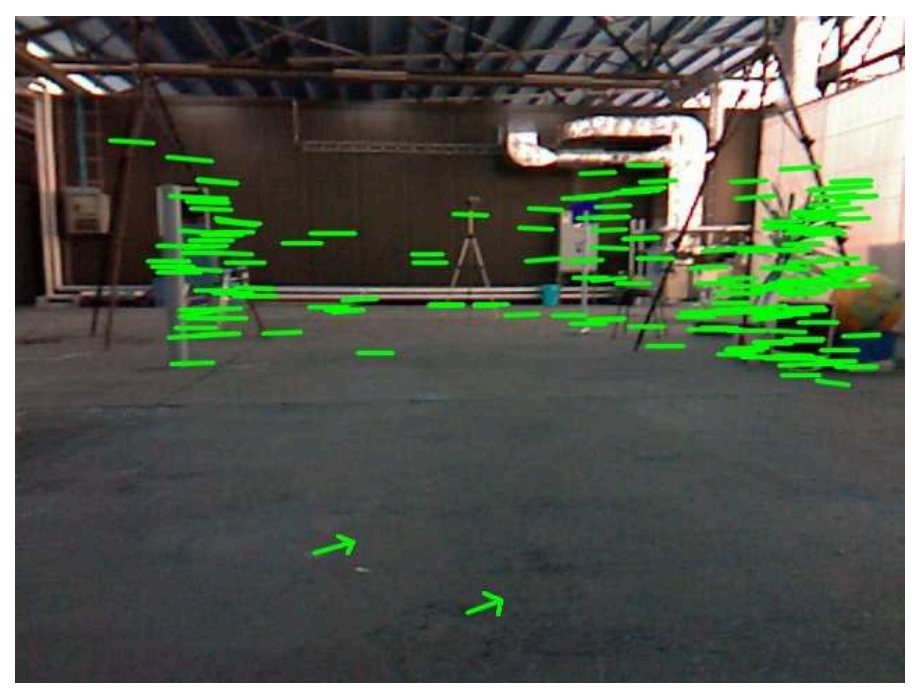

Figure 4.3: Sample image from the Freidburg dataset. The green arrows represent the optical flow generated with the final matches once RANSAC has removed outliers. (the feature detection/descriptor algorithm used was SURF)

As described above, this image also shows the feature flow of the front-end algorithm. The arrow is only displayed on the features on the ground in order to preserve space.

This dataset is much more challenging than the Carleton indoor dataset as this dataset was gathered on-board a robot in an industrial/warehouse setting. This dataset includes motion blur from the RBG sensor, sudden changes in lighting and fast rotations which are not present in the Carleton indoor dataset. However, this dataset contains all the data of the Kinect sensor and hence $20 \mathrm{~Hz}$ of RGB and Depth data is available. To summarize, the Freidburg dataset tests the following features of the algorithm:

1. Kinect sensor mounted onboard a real robot 
2. Vibration from robot motion

3. Sudden changes in lighting as robot drives around warehouse

4. Loop closure as the robot comes back near the initial position

The main computer system employed for this work is a Core i7-2720QM @ 2.2Ghz. The average MIPS of this processor at this speed is 109000 MIPs.

\subsubsection{Implementation details}

The main middle-ware used in this thesis was the Robot Operating System (ROS). The version utilized was ROS Hydro release. In order to acquire the data, the drivers used were the OpenNI drivers. Since the sensor uses two cameras, it is important to ensure that the intensity images and depth images are synchronized. This is important for the case of the Freidburg dataset since there are several cases where the images can be unsynchronized to as much as $20 \mathrm{~ms}$. This can cause serious estimation errors since the depth does not match the motion of the robot. For this reason, the ROS message filter synchronizer class was employed to ensure that the incoming images are at most 1 milisecond apart [78].

\subsubsection{Kinect Sensor Calibration}

As mentioned in the approach section, the Kinect camera and depth sensor values were calibrated using the OpenCV calibration tools [71]. The following table outlines the calibrated values from the Kinect camera utilized for the Carleton indoor dataset: 


\begin{tabular}{|l|l|l|}
\hline Calibration Parameter & IR camera & RGB camera \\
\hline Focal Length & 5.423 & 4.895 \\
\hline $\begin{array}{l}\text { Principal point offset in x (cx) } \\
\text { Principal point offset in y (cy) }\end{array}$ & 0.059 & 0.034 \\
\hline k1 radial coefficent & $1.37 \mathrm{e}-3$ & $-3.57 \mathrm{e}-3$ \\
k2 radial coefficent & $-1.72 \mathrm{e}-4$ & $-4.23 \mathrm{e}-4$ \\
k3 radial coefficent & $-2.61 \mathrm{e}-5$ & $-1.14 \mathrm{e}-6$ \\
\hline T1 tangential coefficent & $-2.56 \mathrm{e}-3$ & $-7.89 \mathrm{e}-5$ \\
T2 tangential coefficent & $5.51 \mathrm{e}-4$ & $-2.19 \mathrm{e}-4$ \\
\hline
\end{tabular}

Table 4.1: Calibration parameters for the infra-red camera RGB camera of the Kinect sensor used in the indoor dataset

The calibration coefficients used in the Freidburg warehouse dataset are available at [77].

\subsubsection{Feature detection/descriptor timing comparison}

As discussed above, the feature detection/descriptor step is the most computationally expensive step. Figure 4.4 shows the feature detection and descriptor times for the algorithms in question using the images captured. 

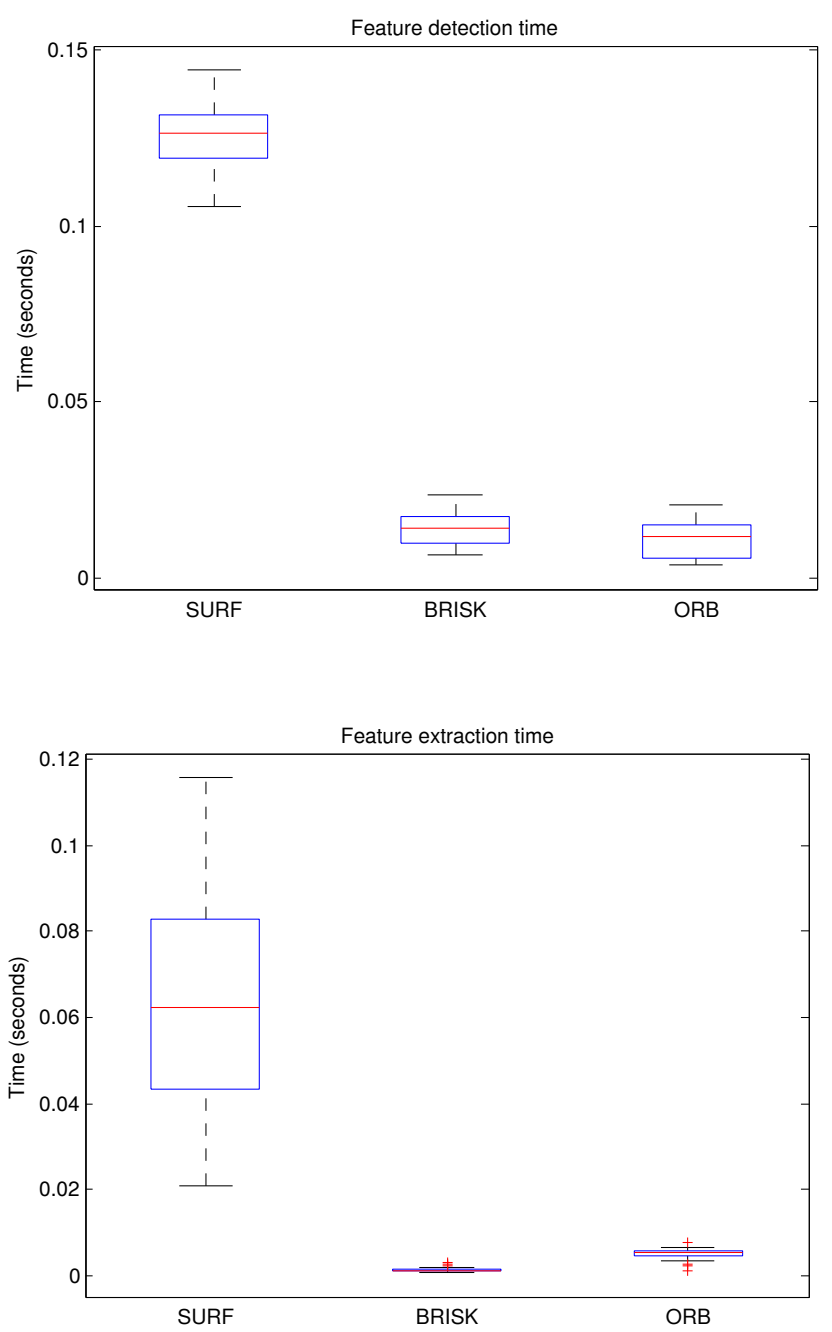

Figure 4.4: Box plot of feature descriptor and detection time for SURF, BRISK and ORB algorithms (Carleton indoor dataset)

As it can be seen in Figure 4.4, SURF is the most time consuming algorithm both in terms of feature detection and descriptor was SURF. The BRISK and ORB algorithms have similar processing times with BRISK being only slightly faster. The feature description in the SURF step was also much more time consuming than that of BRISK and ORB. Since the binary descriptor is part of the feature detection step in BRISK, the time to describe the feature is very small [35] The average times for both the Carleton indoor and Freidburg warehouse datasets are similar. 


\subsubsection{Matching results}

As described in the previous subsection, the matching step for BRISK and ORB uses both the ratio between the closest features and the "minimum distance multiplier" test to ensure that enough matches are present for RANSAC. The results with and without "minimum distance multiplier" test are summarized in Table 4.2.

\begin{tabular}{|l|l|l|}
\hline$\overline{\text { Matches }}$ & $\overline{\text { MinDistMultiplier }}$ & Algorithm \\
\hline 6.2 & 0 & BRISK \\
21.3 & 3.1 & BRISK \\
5.1 & 0 & ORB \\
25.1 & 2.7 & ORB \\
17.5 & n/a & SURF \\
\hline
\end{tabular}

Table 4.2: The effect of introducing the Minimum Distance Multiplier (MinDistMultiplier) when finding the top matches for BRISK and ORB feature description algorithms (Carleton indoor dataset)

In the above table, $\overline{\text { Matches }}$ is the average number of matches, the

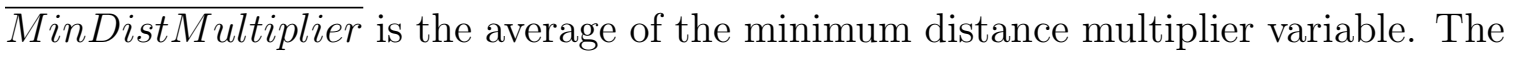
minimum distance multiplier will start at 2.0 and increased by $\Delta=0.5$ as more matches are required. As it can be seen from the table above, the minimum distance multiplier produces more matches that can later be filtered by RANSAC algorithm. Table 4.3 summarizes the results of matching in the Freidburg dataset. 


\begin{tabular}{|l|l|l|}
\hline$\overline{\text { Matches }}$ & $\overline{\text { MinDistMultiplier }}$ & Algorithm \\
\hline 5.3 & 0 & BRISK \\
17.8 & 3.4 & BRISK \\
4.8 & 0 & ORB \\
18.9 & 2.9 & ORB \\
20.4 & n/a & SURF \\
\hline
\end{tabular}

Table 4.3: The effect of introducing the Minimum Distance Multiplier (MinDistMultiplier) when finding the top matches for BRISK and ORB feature description algorithms (Freidburg dataset)

It is also possible to see from the tablet above that the MinDistMultiplier provided enough matches for the Freidburg case as well. It is important to note that the adaptive method did not negatively affected the computation of the matching step since the process of comparing the binary matches is a very fast process.

\subsubsection{Outlier Rejection and transformation refinement per- formance}

This subsection discusses the performance of the transformation estimation algorithm using the standard RANSAC algorithm, the proposed inlier-based RANSAC algorithm and the 2-frame local bundle adjustment refinement. In order to evaluate the error, the widely employed relative pose error (RPE) equation is used [63]. Define the sequence of poses of the estimated trajectory as $P_{1}, P_{2}, P_{3}, \ldots, P_{n} \in S E(3)$ of the motion and the ground truth poses as $G_{1}, G_{2}, G_{3}, \ldots, G_{n} \epsilon S E(3)$. The relative pose error at time $i$ can hence be defined as:

$$
\begin{gathered}
E_{i}=\left(G_{i}^{-1} G_{i+\Delta}\right)^{-1}\left(P_{i}^{-1} P_{i+\Delta}\right) \\
\operatorname{trans} E_{i}=\operatorname{trans}\left(E_{i}\right)
\end{gathered}
$$


where the 'trans' function will extract only the translational error (discarding the rotation error). From a sequence of $\mathrm{n}$ poses, it is possible to obtain $m=n \Delta$ individual relative pose errors. The mean square error (RMSE) from the translation errors can be computed as follows:

$$
R M S E(\operatorname{trans} E)=\sqrt{\left(\frac{1}{m} \sum_{i=1}^{m}\left|\operatorname{trans}\left(E_{i}\right)\right|^{2}\right)}
$$

Using the above equation, it is possible to measure the improvements provided by the various modifications proposed in the work above. Table 4.4 summarizes the results of the test with the different proposed modifications.

\begin{tabular}{|l|l|l|}
\hline Algorithm & RMSE & Descriptor \\
\hline RANSAC & 0.021 & SURF \\
RANSAC+Heur. & 0.019 & SURF \\
RANSAC+Heur.+2F-BA & 0.017 & SURF \\
\hline RANSAC & 0.042 & BRISK \\
RANSAC+Heur. & 0.033 & BRISK \\
RANSAC+Heur.+2F-BA & 0.025 & BRISK \\
\hline RANSAC & 0.045 & ORB \\
RANSAC+Heur. & 0.041 & ORB \\
RANSAC+Heur.+2F-BA & 0.035 & ORB \\
\hline
\end{tabular}

Table 4.4: Accuracy results for the front end system organized by feature descriptor and algorithm variations (Carleton Indoor dataset)

In Table 4.4, the "Heur." implies the addition of the $\delta$ inlier heuristic to RANSAC and "2F-BA" is the two-frame bundle adjustment step. It is important to note that for all descriptors used, the addition of the "Inlier" heuristics improves upon the initial RANSAC estimation. This improvement is certainly more noticeable in the BRISK and ORB descriptors since this cases are more susceptible to large invalid 
transformations. It can also be seen that the two frame bundle adjustment step improves the transformation but not very significantly. Another interesting result is that BRISK actually performed better than ORB in the tests while still keeping the computational load to a similar level. Figure 4.5 displays the average computational time of the whole front end algorithm arranged by featured detector/extractor used:

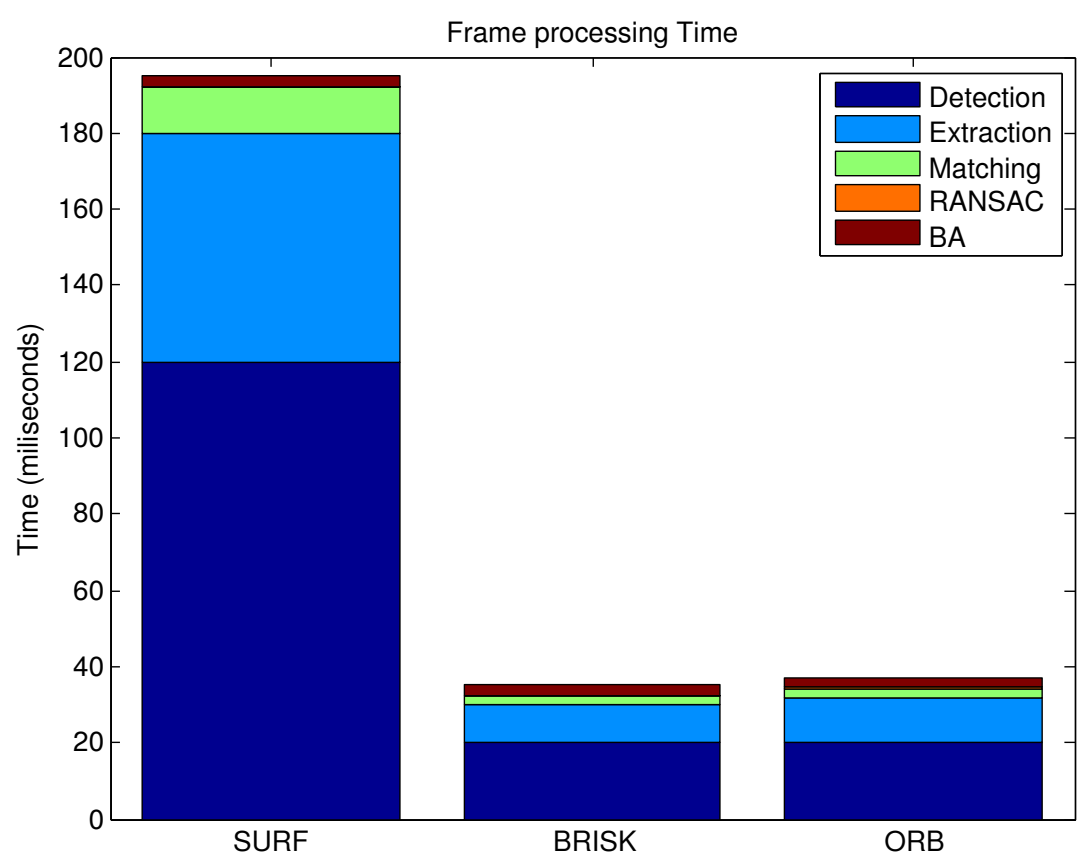

Figure 4.5: Processing time for each frame arranged by feature detection/descriptor algorithm used.

Figure 4.5 shows how the RANSAC and the bundle adjustment step are the smallest processing steps in the algorithm. The RANSAC algorithm took around 0.6 milliseconds for all feature description algorithms. The two frame bundle adjustment step also completed in an average of 2.5 milliseconds. The brute force matching step for the BRISK and ORB algorithms completed in average 2.2 milliseconds while the matching time for the SURF FLANN matching algorithm completed in average 11.5 milliseconds. Although FLANN is generally considered as a very fast matching method [72], this matching still took longer than both ORB and BRISK due to the large dimension of the SURF descriptor. This time study clearly shows how completing the extra Bundle adjustment step is worth the extra computation time as it provides a better estimate for only 2.5 milliseconds of extra processing. Table 4.5 
shows the performance of the front end for the Freidburg dataset:

\begin{tabular}{|l|l|l|}
\hline Algorithm & RMSE & Descriptor \\
\hline RANSAC & 0.044 & SURF \\
RANSAC+Heur. & 0.035 & SURF \\
RANSAC+Heur.+2F-BA & 0.032 & SURF \\
\hline RANSAC & 0.048 & BRISK \\
RANSAC+Heur. & 0.038 & BRISK \\
RANSAC+Heur.+2F-BA & 0.035 & BRISK \\
\hline RANSAC & 0.052 & ORB \\
RANSAC+Heur. & 0.041 & ORB \\
RANSAC+Heur.+2F-BA & 0.037 & ORB \\
\hline
\end{tabular}

Table 4.5: Accuracy results for the front end system organized by feature descriptor and algorithm variations (Freidburg dataset)

As it can be seen in Table 4.5, the performance in the Freidburg dataset is also improved by adding the heuristics and the two-frame bundle adjustment steps. Similarly, SURF performed the best and BRISK and ORB followed in accuracy.

\subsubsection{Back end performance}

The back end system was implemented in another thread than the front end system. Figure 4.6 shows the processing time organized by image number. 


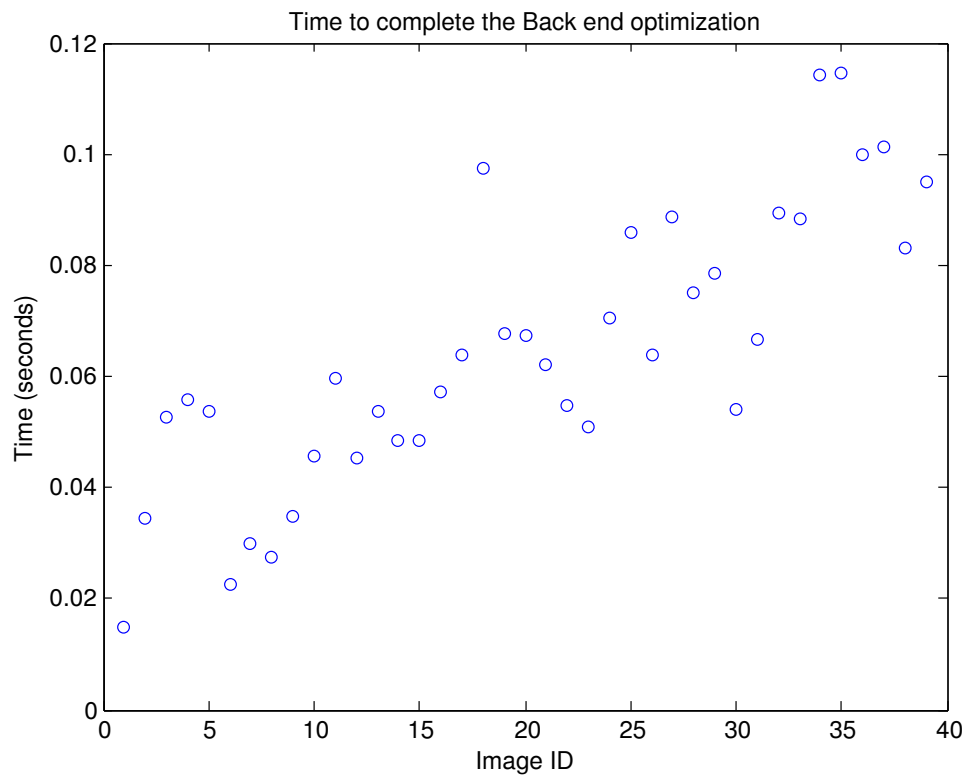

Figure 4.6: Processing time for the back end graph optimization arranged by image number (Carleton indoor Dataset)

As expected, the overall execution time of the back-end grows with the number of poses on the graph. At the end of the optimization, the number of nodes is 40 while the number of edges was 190 (since each node has at most 5 edges). However, the execution time remains close to $100 \mathrm{~ms}$. Figure 4.7 shows the optimization time for the Freidburg dataset 


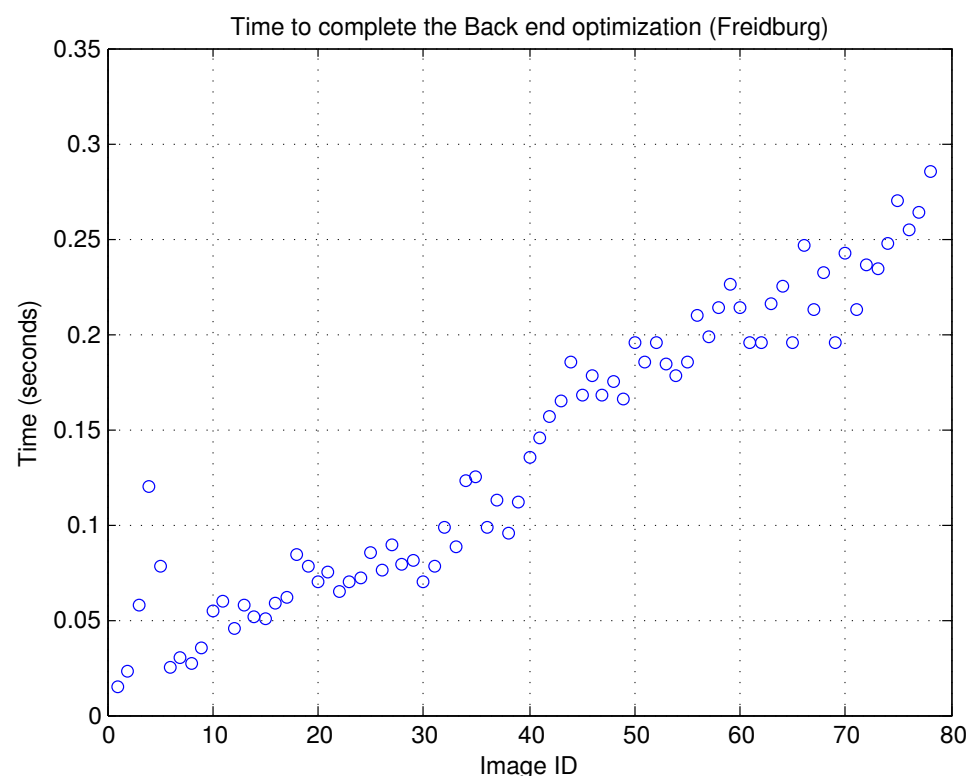

Figure 4.7: Processing time for the back end graph optimization arranged by image number (Freidburg Dataset)

In the case of this dataset, 77 nodes were added to the main graph SLAM tree. The total number of edges was 384 edges and the optimization near the end averaged around $300 \mathrm{~ms}$. In order to evaluate the absolute error, the absolute trajectory error (ATE) equation is used [63]. Define $S$ as the transformation between the ground truth to pose estimation initial offset. In addition, let us define the sequence of poses of the estimated trajectory as $P_{1}, P_{2}, P_{3}, \ldots, P_{n} \in S E(3)$ of the motion and the ground truth trajectory as $G_{1}, G_{2}, G_{3}, \ldots, G_{n} \epsilon S E(3)$. The absolute trajectory error $i$ can hence be defined as:

$$
F_{i}=\left(G_{i}^{-1} S P_{i}\right)
$$

As in the case of the relative pose error, the root mean square error is equal to:

$$
\operatorname{RMSE}\left(F_{1-n}\right)=\operatorname{sqrt}\left(\frac{1}{m} \sum_{i=1}^{m}\left|\operatorname{trans}\left(F_{i}\right)\right|^{2}\right)
$$

The final results organized by feature descriptor algorithm are summarized in Table 4.6. 


\begin{tabular}{|l|l|}
\hline Descriptor & RMSE (ATE) (m) \\
\hline SURF & 0.021 \\
BRISK & 0.027 \\
ORB & 0.035 \\
\hline
\end{tabular}

Table 4.6: Back end SLAM system results organized by feature description algorithm used (Carleton indoor dataset)

It is important to note that for all of the feature description algorithms, the posegraph back end was able to locate loop closure between frames 24 and frame 30 and hence make the connection. In addition, the introduction of the back end reduced the translational error for all feature descriptors as was shown on Table 4.6. Table 4.7 lists the results of applying the full algorithm in the Freidburg dataset. Only the first 65 seconds of the test were employed. It is clear that the position estimation of the system proposed in this thesis is lower than that of the original dataset paper [10]. The original paper achieved an ATE RMSE of 0.02 in the case of the SURF feature extractor. There are several optimizations proposed on [10] paper such as Kinectbased beam measurement model to validate the estimation among others [10]. Since this work attempts to make an algorithm that should operate with stereo cameras or FLASH Lidar, the improvements proposed in [10] cannot be used.

\begin{tabular}{|l|l|}
\hline Descriptor & RMSE (ATE) (m) \\
\hline SURF & 0.025 \\
\hline BRISK & 0.029 \\
\hline ORB & 0.037 \\
\hline
\end{tabular}

Table 4.7: System results organized by feature description algorithm used (Freidburg SLAM dataset)

Figure 4.8 and Figure 4.9 show the results of applying the algorithm on the Freidburg dataset. 


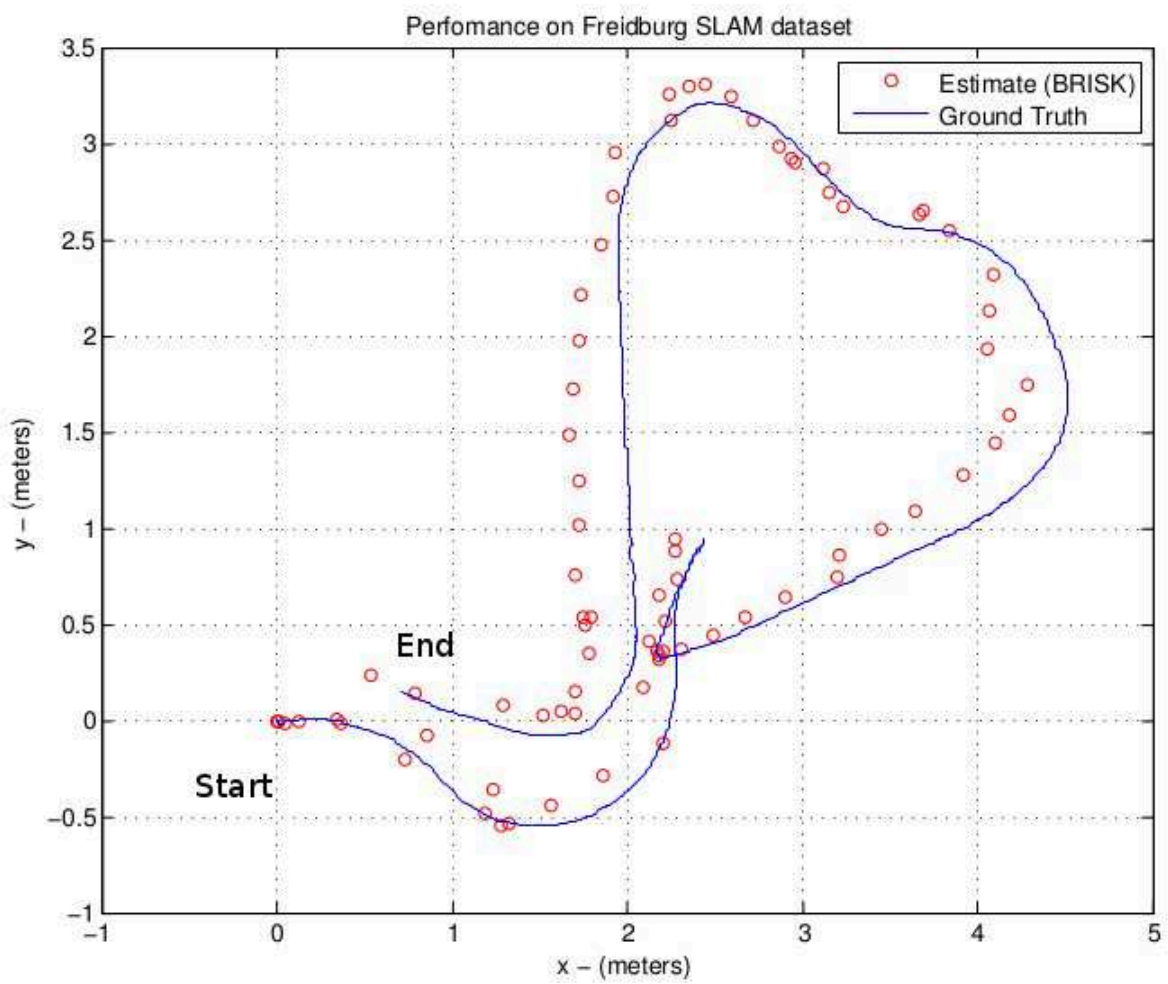

Figure 4.8: Results of applying our approach on the Freidburg SLAM dataset. (The BRISK feature detector was employed) 


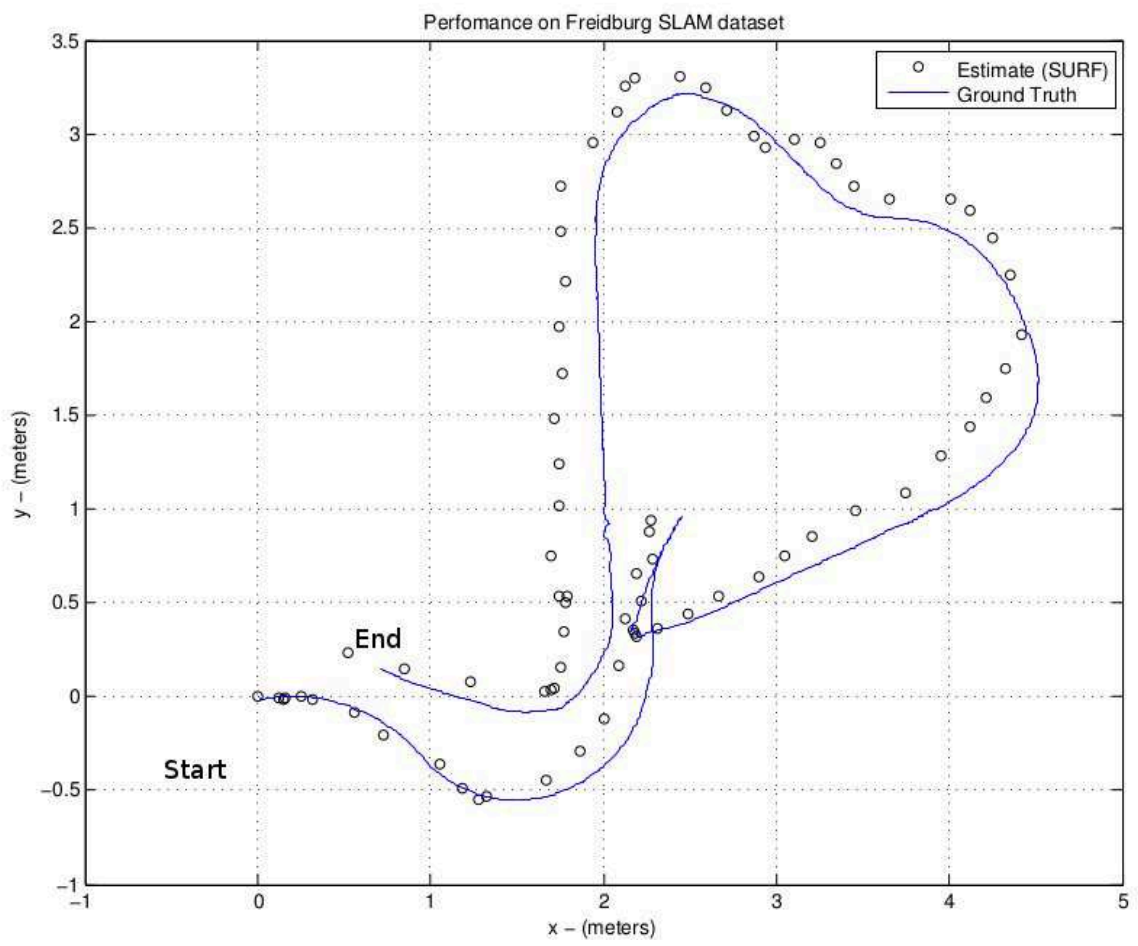

Figure 4.9: Results of applying our approach on the Freidburg SLAM dataset. (The SURF feature detector was employed)

It is important to note that BRISK achieves comparable accuracy on this dataset to the SURF feature descriptor. As it can be seen in Figure 4.8 and Figure 4.9, the robot remains fairly close to the ground truth data (highlited as lines). It can be seen that the robot tends to be bias towards to the left on the last straight line in both 4.8 and Figure 4.9.

The robot path has a more marked left bias on Figure 4.8 near the point $(x=3$, $y=0.5)$ and in the last straight line. The main reason behind this bias is the error in relative estimation of the orientation of the robot. If a certain orientation relative estimation was not correct and slightly biased towards the left, it is likely that the following points will not match the ground truth and be biased towards the left. This error in orientation causes the future estimation points to be biased towards the left. As it can be seen in Figure 4.8, in the point at $(\mathrm{x}=4, \mathrm{y}=2.5)$, the robot pose returns slightly closer to the actual estimated position. This is likely due to another relative estimation error but this time towards the right both in terms of orientation and position error. The error remain relatively constrained until the last straight line in 
Figure 4.8 where the robot again is biased towards the left. Finally, once the robot reaches the loop closure node near point $(\mathrm{x}=0, \mathrm{y}=0)$, the estimation comes much closer to the ground truth robot position.

In the case of Figure 4.9, the left bias is much less marked at the beginning of the traverses. It is interesting to note that both algorithms have a left bias near the point $(\mathrm{x}=3, \mathrm{y}=0.5)$. In the case of Figure 4.9, the robot goes to a right bias nead the point $(\mathrm{x}=4,2.5)$. This is likely due to a sudden rotation at points $(\mathrm{x}=3, \mathrm{y}=0.5)$ and $(\mathrm{x}=4,2.5)$; a sudden left rotation near $(\mathrm{x}=3, \mathrm{y}=0.5)$ and sudden right rotation $(\mathrm{x}=4$, $2.5)$. These rotations likely caused error in the estimation. Similarly to the case with Figure 4.8, the estimation follows the ground truth closely once the robot comes back to the loop closure position $(0,0)$. 


\section{Chapter 5}

\section{Discussion}

\subsection{Results discussion}

This section summarizes the results and discusses their implications with respect to visual SLAM systems for exploration robots.

\subsubsection{Front-end discussion}

As it can be seen from Table 4.4, the heuristics employed in both the matching strategy and the RANSAC algorithm helped to reduce the estimation error for the front- end using BRISK and ORB feature detectors as well as SURF. This heuristics made the BRISK feature detector be capable of producing an RPE RMSE of $3.3 \mathrm{~cm}$. Furthermore, the two-frame bundle adjustment step improved on the intial estimate for all feature detection algorithms. Notably, for the BRISK feature detector, the RPE RMSE was $2.5 \mathrm{~cm}$ after the two frame bundle adjustment. This implies that BRISK was $7 \mathrm{~mm}$ more error than SURF while being 5 times faster to compute.

In the case of the Freidburg dataset, the heuristics and the two frame bundle adjustment also reduced the error and for the BRISK feature detector the final RPE RMSE was $3.5 \mathrm{~cm}$. Unfortunately, the work in [10] did not include RPE results for the front-end part of the algorithm. The main reported error numbers available on the paper use the ATE RMSE metric [10].

It is also possible to compare the performance obtained to other algorithms published. The visual odometer onboard the MER robots is described in detail in this work [1]. Figure 5.1 [1] illustrates the performance of the algorithm when compared to wheel odometry. 


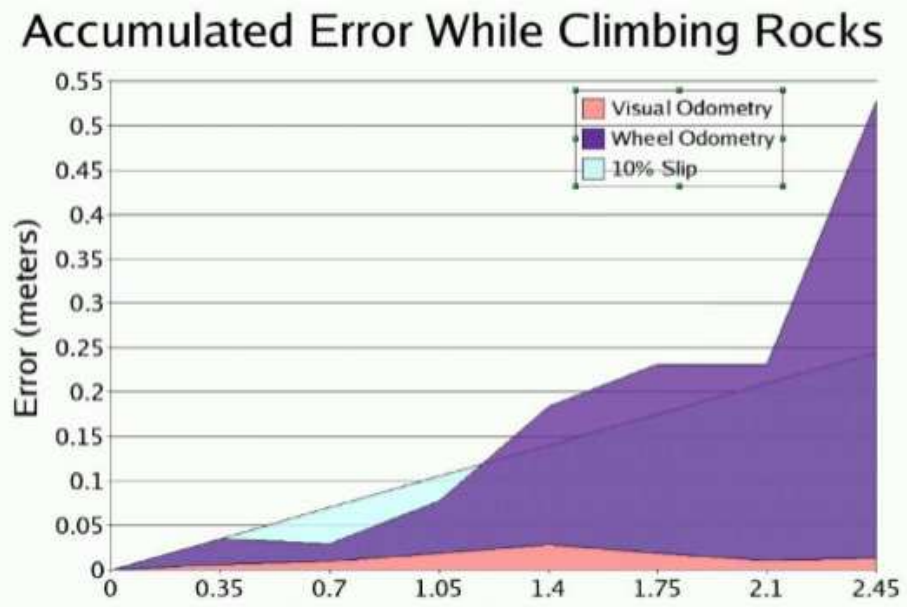

Figure 5.1: Visual Odometry Error measured during a 2.45 meter drive using HAZCAMs on the MER Surface System Testbed Lite rover.

The algorithm described above uses Harris Corners, RANSAC to reject outliers and a motion estimation step uses a statistical estimation problem using robot and feature landmark uncertainty. Unfortunately, the work in [1] does not include detailed error analysis such as the RPE RMSE approached followed in this thesis and hence comparing them directly is not possible. However, the "Accumulated Error" metric provided in Image 5.1 shows that the error in estimation of the visual odometer is around $4 \mathrm{~cm}$ during the traverse. The results obtained using the approached described in this thesis are close to the results presented in [1].

The processing time of the algorithm described in [1] is not discussed in depth in the publication. For this reason, it is also not possible to compare them directly. However, the Harris corner feature detection algorithm used by [1] takes approximately 10ms while BRISK takes approximately 20ms in the computer system used for this thesis. BRISK is a much more stable feature detector and descriptor which should provide better accuracy for tracking and full visual SLAM. The stability of BRISK also can allow for the front-end algorithm to be run less frequently which will therefore decrease the computational load on the system. In addition, the work of [1] keeps track of several features (in the several hundreds magnitude) which cause take longer to computer RANSAC and other matching steps. In this work, a minimum of 20 matches can be employed to complete the outlier rejection and motion estimation steps.

This time comparison shows that it is likely that the algorithm proposed in this 
thesis can be used on a computational constrained system since it takes a similar time to complete as flight versions of the algorithm. Our proposed solution is also considerable faster than proposed desktop versions of visual odometry algorithms [10] [31] [9]

\subsubsection{Back-end discussion}

The back end system included in this thesis achieved loop closure in a traverse of 14.5 meters in under $300 \mathrm{~ms}$ on the computer system in question. While this system attempts to use as little computation as possible, it is necessary to compare the currently added node to at least 5 other nodes as well as completing several checks for the incoming frames. In addition, it is necessary to run the optimization of the whole tree with respect to a reference frame. This computation time truly highlights the difficulty of keeping processing power to a minimum in back-end SLAM systems. The time to complete the optimization of $300 \mathrm{~ms}$ with 80 nodes is not very long when compared to $200 \mathrm{~ms}$ of a single visual odometry processing step of most visual odometry systems.

Most full bundle adjustment algorithms will take much longer to compute the final solution than the solution proposed in this thesis. Most work in structure from motion is designed to run off-line. However, there are several back-end SLAM algorithms designed to run in constant time/limited processing such as [6] [7] (based on relative bundle adjustment) or [79] (sliding window bundle adjustment). The aforementioned algorithms are likely to perform better than the algorithm proposed in this thesis for long traverses.

The results in this thesis are similar in terms of accuracy and timing to those described in [10]. The approach in this thesis was heavily inspired in the work of [10] and therefore the similarities are expected.

\subsubsection{Future Work}

There are several areas where the algorithm proposed can be improved.

1. The RANSAC algorithm employed was 3-point RANSAC. There are novel techniques that reduce the number of RANSAC iterations by using 2-point RANSAC and 1-point RANSAC. These improvements are possible due to the fact that 
the sensor is mounted on a mobile robot where the degrees of freedom are limited [45]. The number of iterations grows exponentially with the number of data points to estimate and hence 1-point RANSAC will require much less processing power when compared to 3-point RANSAC.

2. The RANSAC algorithm employed euclidean distance to compute the error between the transformation proposal and the feature location. This technique was employed for simplicity. In order to incorporate the uncertainty of the measurements, it is possible to employ the Mahalanobis distance as opposed to the Euclidean distance. This approach is followed by several publications [10] [62].

3. It is clear from the experimentation section that running the two-frame bundle adjustment step greatly helps to reduce the error of the front-end estimation. However, several authors use windowed-local bundle adjustment as opposed to just two-frame bundle adjustment [23] [57]. While this step might increase the computational load, it will be interesting to evaluate whether or not the increase in accuracy is worth the computational load.

4. The back-end system presented in this thesis runs on real-time and is relatively inexpensive in terms of computation. However, there is large body of research in the field of relative bundle adjustment [6] [8] [7]. It will be interesting to evaluate relative bundle adjustment in this application. Unfortunately, at the time of this thesis, there is no publicly available implementation of this algorithm and the algorithm itself is rather complex to implement.

5. The algorithm designed and the implementation is highly modular. The software was written so that any subcomponent can be swapped and hence allows for testing of several feature extraction algorithms, matching strategies, outlier rejection, etc. This will allow for easy testing of improvements of the system

6. The algorithm must be tested in an outdoor environment. Although Harris corners and SURF have both been tested in outdoor environments similar to those of space exploration missions [47] [6], BRISK and ORB should be tested outdoor. In addition, the robustness of the whole algorithm will benefit greatly from running some tests outdoors. 


\section{Chapter 6}

\section{Conclusion}

\subsection{Conclusion}

In this thesis, a visual SLAM system for use on computationally constrained environments has been presented. The front-end of the SLAM system evaluated different feature description algorithms with the goal of reducing computation while keeping the accuracy to an acceptable level. The feature descriptor BRISK [35] achieved a RMSE error of $2.3 \mathrm{~cm}$ on a small testing data. The accuracy of BRISK is comparable to that of SURF while being one order of magnitude faster. The RANSAC algorithm was used to estimate the frame-to-frame motion. The test for the best transformation in RANSAC employed both the transformation fit and the number of inliers. This is shown to reduce the transformation estimation error for BRISK and ORB feature descriptors. Once RANSAC has estimated the initial motion, two-frame sparse bundle adjustment is applied to the transformation and the inlier descriptors to further refine the transformation. Finally, the transformation is sent to a back-end pose-graph optimization system to further reduce the global drift and achieve loop closure. The back end system is proven to decrease the pose errors and be capable of achieving loop closure.

\subsubsection{Contributions}

The contributions of this thesis are listed below:

1. This thesis is the first visual SLAM system using BRISK as the main feature detection and description algorithm: The author has been unable to see the new BRISK algorithm being employed in the visual odometry literature. It has 
been proven in this thesis that BRISK achieve similar performance to complex feature descriptors such as SURF. BRISK is an order of magnitude faster to compute when compared to SURF (23ms for BRISK, $220 \mathrm{~ms}$ for SURF). The RMSE translation error of the front-end with SURF was $0.017 \mathrm{~m}$ while the RMSE translation error with BRISK was $0.025 \mathrm{~m}$.

2. This thesis includes a dynamic matching strategy that ensures that enough good matches are passed to the outlier rejection step: For robust feature descriptors such as SURF and SIFT, the "ratio-test" generates enough good matches for transformation estimation in the experiments conducted. In the case of ORB and BRISK the "ratio-test" does not always provide enough good matches. Therefore an adaptive matching strategy introduced a parameter called "Minimum Distance Multiplier" that allows matches that were closer than "minimum Distance Multiplier" multiplied by the Minimum distance of all matches. This parameter will increment if more matches are required. This adaptive method was proven to have no significant impact in the performance of the matching algorithm.

3. This thesis modifies the standard RANSAC algorithm to account for the number of inliers on an specific model instead of only the RMSE error as most implementations. For robust feature descriptors such as SURF and SIFT, the standard RMSE test proved a good test to reject outliers. Since there were many good matches with SURF and SIFT, the best transform had the lowest RMSE error. In the case of ORB and BRISK, it was observed that only using RMSE as test for fit will result in bogus transformations. For this reason, the test for fitness included the number of inliers and the RMSE. This technique will penalize transformation with few inliers which are likely to be bogus transformations. This extra step also increased the accuracy of SURF transformation estimation.

4. This thesis presents a visual SLAM system using only a vision sensor that is designed to run on computationally constrained systems. The visual SLAM system presented runs on real time on a CPU. The front end uses computationally inexpensive feature detection and descriptors while keeping good accuracy. The back-end system uses also a computationally inexpensive graph SLAM implementation that runs on real time and achieves loop closure. 


\section{List of References}

[1] L. Matthies, M. Maimone, A. Johnson, Y. Cheng, R. Willson, C. Villalpando, S. Goldberg, A. Huertas, A. Stein, and A. Angelova, "Computer Vision on Mars," International Journal of Computer Vision, vol. 75, no. 1, pp. 67-92, Mar. 2007. [Online]. Available: http://link.springer.com/10.1007/s11263-007-0046-z

[2] B. K. Cooper, F. R. Hartman, P. C. Leger, M. W. Maimone, S. a. Maxwell, A. Trebi-ollennu, E. W. Tunstel, and J. R. Wright, "Mars Exploration Rover Surface Operations," IEEE Robotics and Automation Magazine, no. 1070-9932, pp. 63-71, 2006.

[3] C. F. Olson, L. H. Matthies, M. Schoppers, and M. W. Maimone, "Rover navigation using stereo ego-motion," Robotics and Autonomous Systems, vol. 43, pp. 215-229, 2003.

[4] M. Bajracharya, M. W. Maimone, and D. Helmick, "Autonomy for Mars Rovers: Past, present, and future," IEEE Magazine, vol. 41, pp. 44-50, 2008.

[5] G. B. Sanders, N. Jsc, C.-c. M. Duke, D. Linne, K. Sacksteder, S. Nozette, D. Rapp, M. Downey, D. Mckay, K. Romig, R. Johnson, W. Larson, P. Curreri, E. Mccullough, E. Rice, L. Clark, L. Martin, R. Zubrin, and T. Simon, "NASA In-Situ Resource Utilization ( ISRU ) Capability Roadmap Final Report," Moon, pp. 1-49, 2005.

[6] P. Furgale, "Bundle Adjustment - A computational efficient solution for feature-based batch SLAM," UTAIS, Toronto, ON, Tech. Rep., 2010. [Online]. Available: http://www.mathworks.com/matlabcentral/fileexchange/44031

[7] D. Sibley and C. Mei, "Adaptive relative bundle adjustment." Robotics: science and systems, vol. 220, no. 3, pp. 1-8, 2009.

[8] C. Mei, G. Sibley, M. Cummins, P. Newman, and I. Reid, "RSLAM: A System for Large-Scale Mapping in Constant-Time Using Stereo," International Journal of Computer Vision, vol. 94, no. 2, pp. 198-214, Jun. 2010. [Online]. Available: http://link.springer.com/10.1007/s11263-010-0361-7

[9] A. S. Huang, A. Bachrach, P. Henry, M. Krainin, D. Fox, and N. Roy, "Visual Odometry and Mapping for Autonomous Flight Using an RGB-D Camera," Massachusetts Institute of Technology, Cambridge, MA, Tech. Rep., 2008. 
[10] F. Endres, J. Hess, J. Sturm, D. Cremers, and W. Burgard, "3D Mapping with an RGB-D Camera," IEEE Transactions on Robotics, vol. 30, no. 1, pp. 1-11, 2014.

[11] A. J. Davison, I. D. Reid, N. D. Molton, and O. Stasse, "MonoSLAM: real-time single camera SLAM." IEEE transactions on pattern analysis and machine intelligence, vol. 29, no. 6, pp. 1052-67, Jun. 2007. [Online]. Available: http://www.ncbi.nlm.nih.gov/pubmed/17431302

[12] G. Klein and D. Murray, "Parallel Tracking and Mapping for Small AR Workspaces," in 6th IEEE and ACM International Symposium on Mixed and Augmented Reality. IEEE - International Symposium on Mixed and Augmented Reality, Nov. 2007, pp. 1-10. [Online]. Available: http://ieeexplore.ieee.org/lpdocs/epic03/wrapper.htm?arnumber=4538852

[13] Charles E. Dunn, John J. Lenz and J. L. Sweeney, "Gemini Familiarization Manual," McDonnel Corporation, Owego, NY, Tech. Rep., 1968.

[14] A. Kent, "Computers in Spaceflight: The NASA Experience," NASA JPL, La Canada Frintidge, CA, Tech. Rep., 1987.

[15] Department of Defence USA, "Test Method Standard - Microcircuits - MILSTD-883E," Department of Defence USA, Columbus OH, Tech. Rep., 1996.

[16] Intel Corporation, "Intel core i5-2550K Processor information page," pp. 1-3, 2008. [Online]. Available: http://ark.intel.com/products/65647

[17] G. Lentaris, D. Diamantopoulos, K. Siozios, I. Stamoulias, and I. Kostavelis, "SPARTAN : Efficient Implementation of Computer Vision Algorithms for Autonomous Rover Navigation," pp. 1-10.

[18] T. G. M.R. Andersen, T. Jensen, P. Lisouski, A.K. Mortensen, M.K. Hansen and P. Ahrendt, "Kinect Depth Sensor, Evaluation for Computer vision applications," AARHUS University - Department of Engineering, Aarthus, Denmark, Tech. Rep., 2012.

[19] P. Grey, "Point Grey - Bumblebee2 fact sheet," Vancouver, Canada, pp. 1-0, 2008. [Online]. Available: http://www.ptgrey.com/ bumblebee2-firewire-stereo-vision-camera-systems

[20] Advance Scientific, "Peregrine 3D Flash LIDAR Vision System," pp. 110, 2014. [Online]. Available: http://www.advancedscientificconcepts.com/ products/Peregrine.html

[21] H. Durrant-whyte and T. Bailey, "Simultaneous Localisation and Mapping ( SLAM ): Part I: The Essential Algorithms," IEEE Robotics and Automation Magazine, pp. 1-9, 2006.

[22] D. Scaramuzza and F. Fraundorfer, "Visual Odometry - The first 30 years and fundamentals," IEEE Robotics and Automation Magazine, Tech. Rep. December, 2011. 
[23] — _ - "Visual Odometry: Part II: Matching, Robustness, Optimization and Applications," IEEE Robotics and Automation Magazine, no. June, pp. 78-90, 2012.

[24] H. P. Moravec, "Obstacle avoidance and navigation in the real world by a seeing robot rover," Robotics Institute, Carnegie Mellon University, Tech. Rep., 1980.

[25] L. Matthies, "Stereo vision for planetary rovers: Stochastic modeling to near real-time implementation," International Journal of Computer Vision, vol. 8, no. 1, pp. 71-91, Jul. 1992. [Online]. Available: http: //link.springer.com/10.1007/BF00126401

[26] D. Scaramuzza, "Omnidirectional vision: From camera calibration to robot motion estimation," Doctor of Science, university of Zurich, 2008.

[27] G. Bradski, "OpenCV Library," Dr. Dobb's Journal of Software Tools, no. 2236121, 2008.

[28] E. Rosten, R. Porter, and T. Drummond, "Faster and better: a machine learning approach to corner detection," Tech. Rep., Oct. 2008. [Online]. Available: http://arxiv.org/abs/0810.2434

[29] C. Harris and M. Stephens, "A Combined Corner and Edge Detector," Procedings of the Alvey Vision Conference 1988, pp. 23.1-23.6, 1988. [Online]. Available: http://www.bmva.org/bmvc/1988/avc-88-023.html

[30] C. T. Jianbo Shi, "Good Features to Track," in IEEE Conference on Computer Vision and Pattern Recognition. Seatle, Washington, USA: IEEE, 1994, pp. 593 -600 .

[31] a. Howard, "Real-time stereo visual odometry for autonomous ground vehicles," 2008 IEEE/RSJ International Conference on Intelligent Robots and Systems, pp. 3946-3952, Sep. 2008. [Online]. Available: http: //ieeexplore.ieee.org/lpdocs/epic03/wrapper.htm?arnumber=4651147

[32] G. Lowe, "Distinctive image features from scale-invariant keypoints," Computer and Information Science, vol. 60, no. 2(2004), pp. 91-110, 2004.

[33] H. Bay, T. Tuytelaars, and L. V. Gool, "SURF : Speeded Up Robust Features," Computer Vision and Image Understanding, vol. 110, no. 3, pp. 346-359, 2008.

[34] E. Rublee and G. Bradski, "ORB : an efficient alternative to SIFT or SURF," in IEEE International Conference on Computer Vision. Barcelona, Spain: IEEE, 2011, pp. $2564-2571$.

[35] S. Leutenegger, M. Chli, and R. Y. Siegwart, "BRISK : Binary Robust Invariant Scalable Keypoints," in IEEE International Conference on Computer Vision. Barcelona, Spain: IEEE, 2011, pp. 2548 - 2555.

[36] M. Calonder, V. Lepetit, C. Strecha, and P. Fua, "Brief Binary Robust Independent Elementary Features," in Proceedings of the 11th European conference on Computer vision. Berlin, Germany: ACM, 2010, pp. 778-792. 
[37] M. Agrawal, K. Konolige, and M. R. Blas, "CenSurE: Center surround extremas for realtime feature detection and matching," Lecture Notes in Computer Science (including subseries Lecture Notes in Artificial Intelligence and Lecture Notes in Bioinformatics), vol. 5305 LNCS, pp. 102-115, 2008.

[38] A. Schmidt and M. Kraft, "Comparative Assessment of Point Feature Detectors and Descriptors in the Context of Robot Navigation," Journal of Automation, Mobile Robotics 85 Intelligent Systems, vol. 7, pp. 11-20, 2013.

[39] R. W. Rafael Gonzales, Digital Image processing (3rd Edition), 3rd ed., 2007.

[40] M. Muja and D. G. Lowe, "Fast Approximate Nearest Neighbors with Automatic Algorithm Configuration," International Conference on Computer Vision Theory and Applications (VISAPP '09), pp. 1-10, 2009. [Online]. Available: papers2://publication/uuid/3C5A483A-ADCA-4121-A768-8E31BB293A4D

[41] P. Indyk and A. Andoni, "Near-Optimal Hashing Algorithms for Approximate Nearest Neighbor in High Dimensions $\backslash$ n47th IEEE Symposium on Foundations of Computer Science," vol. 51, no. 1, pp. 117-122, 2006. [Online]. Available: http://doi.ieeecomputersociety.org/10.1109/FOCS.2006.49

[42] M. Muja, "FLANN - Fast Library for Approximate Nearest Neighbors." [Online]. Available: http://www.cs.ubc.ca/research/flann/

[43] B. K. Horn and B. Schunck, "Determining optical flow: a retrospective," Artificial Intelligence, vol. 59, pp. 81-87, 1993.

[44] R. C. B. Martin A. Fischler, "Random Sample Consensus : A Paradigm for Model Fitting with applications to image analysis and automated Cartography," Communications of the ACM, pp. 381-395, 1980.

[45] D. Scaramuzza, F. Fraundorfer, and R. Siegwart, "Real-time monocular visual odometry for on-road vehicles with 1-point RANSAC," in 2009 IEEE International Conference on Robotics and Automation. Kobe: IEEE, 2009, pp. $4293-$ 4299.

[46] L. Matthies and S. Shafer, "Error modeling in stereo navigation," IEEE Journal on Robotics and Automation, vol. 3, 1987.

[47] M. Maimone, Y. Cheng, and L. Matthies, "Two Years of Visual Odometry on the Mars Exploration Rovers," NASA JPL, Pasadena, CA, USA, Tech. Rep., 2006 .

[48] K. Konolige, M. Agrawal, and J. Sola, "Large scale visual odometry for rough terrain," Proc International Symposium on Robotics Research, vol. 2, pp. 1150-1157, 2007. [Online]. Available: http://citeseerx.ist.psu.edu/viewdoc/ download?doi=10.1.1.72.1240\&amp;rep=rep1\&amp;type=pdf

[49] J. Engel, T. Sch, and D. Cremers, "LSD-SLAM: Large-Scale Direct Monocular SLAM," Eccv, pp. 1-16, 2014. 
[50] S. J. Julier and W. Dc, "Counter Example t o the Theory of Simultaneous Localization and Map Building," in IEEE International Conference on Robotics and Automation, no. Ic, 2001, pp. 4238-4243.

[51] J. Guivant, J. Guivant, E. Nebot, and E. Nebot, "Optimization of the Simultaneous Localization and Map Building Algorithm for Real Time Implementation," IEEE Transactions on Robotics and Automation, vol. 17, pp. 242-257, 2001.

[52] A. Monjazeb, "Autonomous mobile robot positioning using Unscented HybridSLAM," PhD, Carleton University, 2013. [Online]. Available: http: //ieeexplore.ieee.org/xpls/abs_all.jsp?arnumber $=6669976$

[53] T. Bailey and H. Durrant-Whyte, "Simultaneous localization and mapping (SLAM): Part II," IEEE Robotics and Automation Magazine, vol. 13, pp. 108117, 2006.

[54] R. Eustice, M. Walter, and J. Leonard, "Sparse extended information filters: Insights into sparsification," 2005 IEEE/RSJ International Conference on Intelligent Robots and Systems, IROS, pp. 641-648, 2005.

[55] K. Ni, D. Steedly, and F. Dellaert, "Tectonic SAM: Exact, out-of-core, submapbased SLAM," Proceedings - IEEE International Conference on Robotics and Automation, pp. 1678-1685, 2007.

[56] G. Grisetti, R. Kummerle, C. Stachniss, and W. Burgard, "A Tutorial on Graph-Based SLAM," IEEE Intelligent transportation system council, pp. 31-43, 2010. [Online]. Available: http://www2.informatik.uni-freiburg.de/ stachnis/ pdf/grisetti10titsmag.pdf

[57] B. Triggs, P. McLauchlan, R. Hartley, and A. Fitzgibbon, "Bundle Adjustment - A Modern Synthesis," Vision Algorithms: Theory and Practice, vol. 1883, pp. 298-372, 2000. [Online]. Available: http://dx.doi.org/10.1007/3-540-44480-7_21

[58] K. Konolige, "Sparse Bundle Adjustment," Procedings of the British Machine Vision Conference 2010, pp. 102.1-102.11, 2010. [Online]. Available: http://www.bmva.org/bmvc/2010/conference/paper102/index.html

[59] E. Rosten and T. Drummond, "Machine learning for high-speed corner detection," in European Conference on Computer Vision, 2006, pp. 1-14.

[60] S. Rusinkiewicz and M. Levoy, "Efficient variants of the ICP algorithm," Proceedings Third International Conference on 3-D Digital Imaging and Modeling, pp. 145-152. [Online]. Available: http://ieeexplore.ieee.org/lpdocs/ epic03/wrapper $\cdot$ htm? arnumber $=924423$

[61] F. Pomerleau, F. Colas, R. Siegwart, and S. Magnenat, "Comparing ICP variants on real-world data sets," Autonomous Robots, vol. 34, no. 3, pp. 133-148, Feb. 2013. [Online]. Available: http://link.springer.com/10.1007/s10514-013-9327-2

[62] P. Henry, M. Krainin, E. Herbst, X. Ren, and D. Fox, "RGB-D Mapping : Using Depth Cameras for Dense 3D Modeling of Indoor Environments," in In RGB-D: Advanced Reasoning with Depth Cameras Workshop in conjunction with RSS, Berlin, Germany, 2012. 
[63] F. Endres, J. Hess, N. Engelhard, J. Sturm, D. Cremers, and W. Burgard, "An evaluation of the RGB-D SLAM system," 2012 IEEE International Conference on Robotics and Automation, vol. 3, no. c, pp. 1691-1696, May 2012. [Online]. Available: http://ieeexplore.ieee.org/lpdocs/epic03/wrapper. htm?arnumber $=6225199$

[64] F. Steinbrücker, "Real-Time Visual Odometry from Dense RGB-D Images," Department of Computer Science, Technical University of Munich, Munich, Germany, Tech. Rep. 1, 2009.

[65] R. Kummerle, G. Grisetti, H. Strasdat, K. Konolige, and W. Burgard, "G2o: A general framework for graph optimization," in 2011 IEEE International Conference on Robotics and Automation. Shangai: Ieee, May 2011, pp. 3607 - 3613. [Online]. Available: http://ieeexplore.ieee.org/lpdocs/epic03/wrapper. htm?arnumber $=5979949$

[66] M. I. a. Lourakis and A. a. Argyros, "SBA: A software Package for Gewneric Sparse Bundle Adjustment," ACM Transactions on Mathematical Software, vol. 36, no. 1, pp. 1-30, 2009.

[67] U. Frese, "A proof for the approximate sparsity of SLAM information matrices," Proceedings - IEEE International Conference on Robotics and Automation, vol. 2005, no. April, pp. 329-335, 2005.

[68] F. M. Mathieu Labbe, "Appearance-Based Loop Closure Detection for online Large-Scale and Long-Term Operation," IEEE Transactions on Robotics, vol. 29, no. 3, pp. $734-745,2013$.

[69] G. Grisetti, S. Grzonka, C. Stachniss, P. Pfaff, and W. Burgard, "Efficient estimation of accurate maximum likelihood maps in 3D," IEEE International Conference on Intelligent Robots and Systems, no. Ml, pp. 3472-3478, 2007.

[70] K. Khoshelham and S. O. Elberink, "Accuracy and resolution of kinect depth data for indoor mapping applications," Sensors, vol. 12, pp. 1437-1454, 2012.

[71] OpenCV, "Camera calibration With OpenCV," 2011. [Online]. Available: http://docs.opencv.org/modules/calib3d/doc/camera_calibration_and_3d_ reconstruction.html

[72] M. Muja and D. G. Lowe, "Fast Matching of Binary Features," 2012 Ninth Conference on Computer and Robot Vision, pp. 404-410, May 2012. [Online]. Available: http://ieeexplore.ieee.org/lpdocs/epic03/wrapper.htm?arnumber= 6233169

[73] K. Mitra and R. Chellappa, "A scalable projective bundle adjustment algorithm using the L norm," Proceedings - 6th Indian Conference on Computer Vision, Graphics and Image Processing, ICVGIP 2008, pp. 79-86, 2008.

[74] N. Carlevaris-bianco, S. Member, M. Kaess, R. M. Eustice, and S. Member, "Generic Node Removal for Factor-Graph SLAM," IEEE Transactions on Robotics, vol. 30, no. 6, pp. 1371 - 1385, 2014. 
[75] M. Cummins and P. Newman, "FAB-MAP: Probabilistic Localization and Mapping in the Space of Appearance," The International Journal of Robotics Research, vol. 27, no. 6, pp. 647-665, Jun. 2008. [Online]. Available: http://ijr.sagepub.com/cgi/doi/10.1177/0278364908090961

[76] M. Cummins, "Highly Scalable Appearance-Only SLAM FABMAP 2.0," The International Journal of Robotics Research, pp. 1-8, 2011.

[77] J. Sturm, N. Engelhard, F. Endres, W. Burgard, and D. Cremers, "A benchmark for the evaluation of RGB-D SLAM systems," IEEE International Conference on Intelligent Robots and Systems, pp. 573-580, 2012.

[78] J. Faust, "ROS wiki - Image Message Filters," pp. 1-2, 2009. [Online]. Available: http://wiki.ros.org/message_filters

[79] H. Strasdat and A. Davison, "Double window optimisation for constant time visual SLAM," IEEE International Conference on Computer Vision, 2011. [Online]. Available: http://ieeexplore.ieee.org/xpls/abs_all.jsp?arnumber= 6126517 


\section{Appendix A}

\section{Sample images from Indoor Carleton Dataset}

The following images provide an illustration of the Carleton dataset and the feature flow when testing the algorithm using the SURF feature detection and description algorithm.
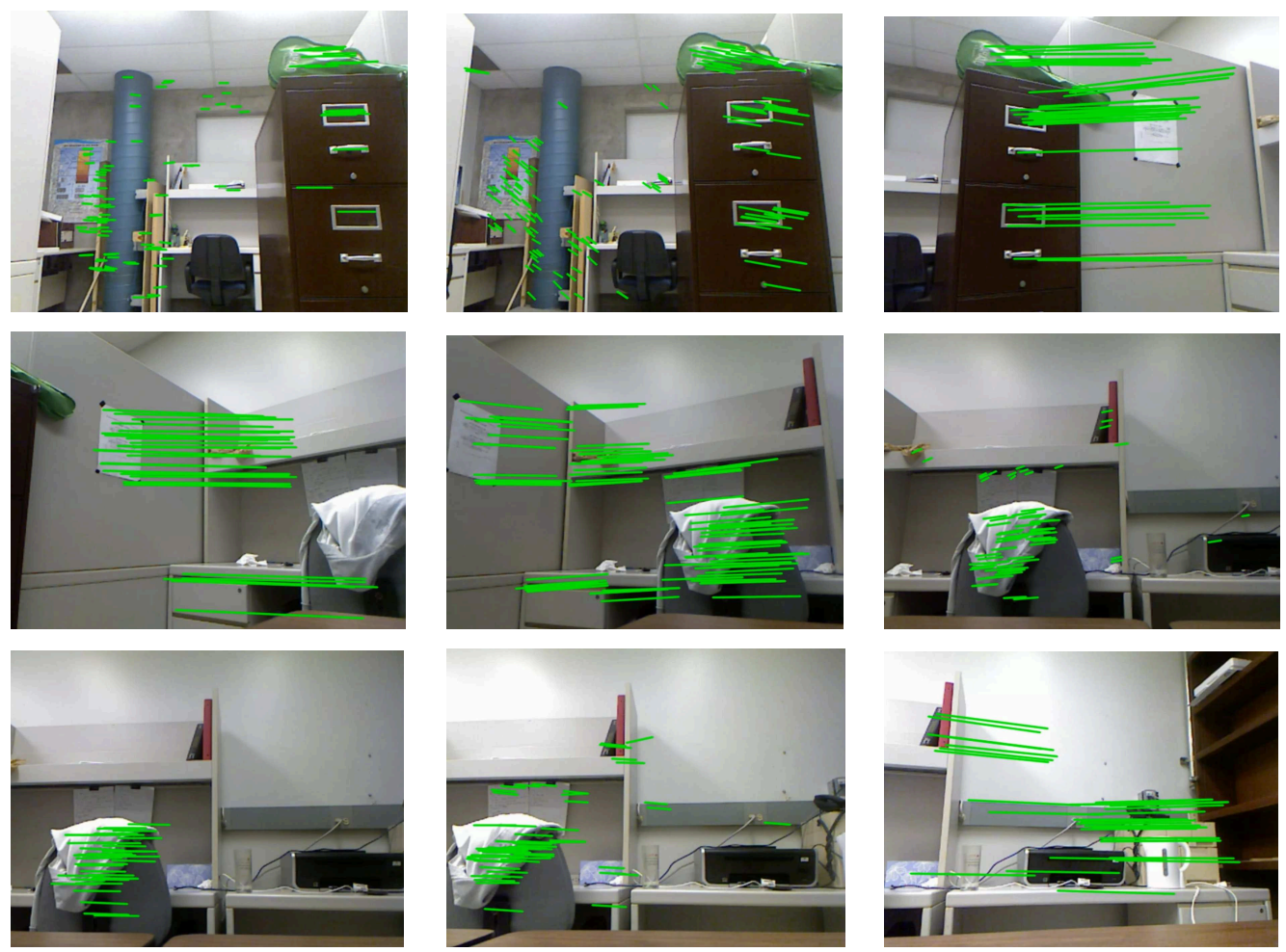